Sep. 8, 2021

\title{
The function of $\alpha$-Catenin mechanosensing in tissue morphogenesis
}

$5 \quad$ Luka Sheppard $^{1}$ and Ulrich Tepass ${ }^{1 *}$

(1) Department of Cell and Systems Biology, University of Toronto; Toronto, ON M5S 3G5, Canada

10

* Corresponding author

Correspondence should be addressed to:

Ulrich Tepass

ORCID: 0000-0002-7494-5942

Department of Cell and Systems Biology

University of Toronto

25 Harbord Street

Toronto, Ontario M5S 2M6

Canada

Phone (all authors): 416-978-5712

\section{ORCID IDs}

LS: 0000-0001-7311-6854

UT: 0000-0002-7494-5942

\section{Emails}

luka.sheppard@mail.utoronto.ca

u.tepass@utoronto.ca 


\begin{abstract}
$\alpha$-catenin couples the cadherin-catenin complex to the actin cytoskeleton. The mechanosensitive $\alpha$-catenin $\mathrm{M}$ region undergoes conformational changes upon application of force to recruit binding partners. Here, we took advantage to the tension landscape in the Drosophila embryo to define three different states of $\alpha$-catenin mechanosensing in support of cell adhesion. Low, medium, and high tension contacts showed $\alpha$-catenin $M$ region-dependent low, medium, and high levels of Vinculin and Ajuba recruitment. In contrast, Afadin/Canoe acts in parallel to $\alpha$-catenin at bicellular

25 low and medium tension junctions, but requires an interaction with $\alpha$-catenin for its tensionsensitive enrichment at high-tension tricellular junctions. Individual $\mathrm{M}$ region domains make complex contributions to cell adhesion through their impact on binding partner recruitment, and redundancies with the function of Afadin/Canoe. Our data argue that $\alpha$-catenin and its interaction partners are part of a cooperative and partially redundant, mechanoresponsive network that supports AJs remodelling during morphogenesis.
\end{abstract}

Short title: $\alpha$-catenin mechanosensing in tissue morphogenesis 


\section{Introduction}

Adherens junctions (AJs) resist actomyosin generated forces to change cell and tissue shape while maintaining tissue cohesion. Dynamic AJs are disassembled and rebuilt in concert with cell contact changes during tissue morphogenesis. Combining both dynamic and stable aspects of AJs requires the interplay of a complex molecular machinery. Significant progress has been made in our understanding of the molecular architecture of AJs since the original discovery of cadherin adhesion molecules (Harris and Tepass, 2010; Maître and Heisenberg, 2013; Takeichi, 2014; 2018; Pinheiro and Bellaïche, 2018). However, much remains to be learned about how AJs operate during specific morphogenetic movements and in particular how AJs associate with the actin cytoskeleton to support cell shape changes and cell movements in tissues such as epithelia.

As tissues adopt new forms during morphogenesis, cell contacts are subjected to contractile forces to elicit coordinated cell shape changes and cell rearrangements. The ability of AJ components to respond to force is believed to be key to maintaining cohesion and tissue integrity during the cellcell contact changes that underpin morphogenesis (Lecuit and Yap, 2015; Ladoux et al., 2015; Pinheiro and Bellaïche, 2018; Charras and Yap, 2018; Clarke and Martin, 2021). The cadherincatenin complex (CCC) physically couples the cytoskeleton of neighboring cells and in response to force - generated often by actomyosin contraction — strengthens adhesive interactions (Le Duc et al., 2010; Yonemura et al., 2010). Extracellular cadherin domains experience force-induced conformation changes, increasing the strength of trans-interactions between cadherins of neighboring cells (Leckband and De Rooij, 2014; Pinheiro and Bellaïche, 2018). The transmission of force through cadherins and the integrity of AJs requires the intracellular physical link to the actin cytoskeleton provided by $\alpha$-catenin (Rimm et al., 1995; Desai et al., 2013; Buckley et al., 2014). $\alpha$-catenin is thought to be the central mechanosensor of the CCC (Yonemura et al., 2010; Ishiyama and Ikura, 2012; Leckband and De Rooij, 2014; Ladoux et al., 2015; Angulo-Urarte et al., 2020). Under the application of force, conformational changes within $\alpha$-catenin lead to enhanced actin binding, the downstream regulation of actin networks, and recruitment of additional AJ components (Yonemura et al., 2010; Choi et al., 2012; Rangarajan and Izard, 2012; Twiss et al., 2012; Huveneers et al., 2012; Barry et al., 2014; Mège and Ishiyama, 2017; Ishiyama et al., 2018; Sarpal et al., 2019; Alégot et al., 2019). Mutational analysis of $\alpha$-catenin has demonstrated its essential role for epithelial cell adhesion similar to E-cadherin (Ecad) and $\beta$ - 
catenin (Armadillo [Arm] in Drosophila) in a number of animal species and diverse tissues (Torres et al., 1997; Kofron et al., 1997; Costa et al., 1998; Schepis et al., 2012; Sarpal et al., 2019; Clarke et al., 2019). However, the function of $\alpha$-catenin mechanosensing in tissue morphogenesis remains largely unexplored.

70

The two mechanosensory domains of $\alpha$-catenin are the central $\mathrm{M}$ region and the $\mathrm{C}$-terminal actinbinding domain (ABD) (Fig. 1A,B). $\alpha$-catenin directly binds F-actin through the ABD as a catchbond, whereby under increasing tension, the strength of this bond, the bond lifetime, increases up to a threshold (Buckley et al., 2014). Force-induced conformational change in the ABD enhances direct F-actin binding (Ishiyama et al., 2018; Xu et al., 2020). In contrast, force-induced conformational changes in the central $\mathrm{M}$ region of $\alpha$-catenin cause the recruitment of other F-actin binding proteins, such as Vinculin and Ajuba, which is thought to reinforce adhesion (Mège and Ishiyama, 2017; Angulo-Urarte et al., 2020).

Here we assessed the function of the $M$ region and its three domains (M1, M2, and M3) in embryonic morphogenesis. M1, M2 and M3 consist of $\alpha$-helical bundles that without tension form a closed conformation supported by a dynamic electrostatic network produced by salt-bridges between these bundles (Ishiyama et al., 2013; Li et al., 2015). Application of tension breaks the salt bridges, exposing cryptic binding sites (Fig. 1A,B). The M1 domain unfurls and binds Vinculin

85 (Vinc), recruiting Vinc to the cell membrane (Yonemura et al., 2010; Ishiyama et al., 2013; Barry et al., 2014; Yao et al., 2014; Kim et al., 2015; Maki et al., 2016; Seddiki et al., 2018). Moreover, the junctional recruitment of Ajuba (Jub) is regulated by mechanosensing through the M region, with M1 preventing Jub recruitment to $\alpha$-catenin under low tension (Alégot et al., 2019; Sarpal et al., 2019). A second conformational change in the M region was reported for the M2 and M3

90 domains that may involve an increase in the angle between M2 and M3 or the partial or complete unfurling of these $\alpha$-helical bundles, which could impact the interaction between $\alpha$-catenin and Afadin (Pokutta et al., 2002; Ishiyama et al., 2013; Yao et al., 2014; Li et al., 2015; Matsuzawa et al., 2018; Sakakibara et al., 2020).

95 We examined the role of the $M$ region in supporting adhesion during Drosophila embryonic morphogenesis, and how the M region cooperates with the $\alpha$-Catenin ( $\alpha$-Cat) interactors Vinc, Jub, 
and Canoe (Cno, the Drosophila ortholog of Afadin) in maintaining tissue integrity as cells undergo shape changes and rearrangements. Contrary to expectations, our recent work has shown that an $\alpha$-Cat constructs which lack the entire $M$ region can fully replace endogenous $\alpha$-Cat in the 100 development of the wing disc epithelium (Sarpal et al., 2019). Deletion of individual M region domains could also support normal epithelial integrity of the disc epithelium, and the ovarian follicular epithelium (Desai et al., 2013; Sarpal et al., 2019). However, deletion of M1 interfered with the normal regulation of tissue growth. Tension sensing through M1 is required to prevent the constitutive recruitment of Jub to $\alpha$-Cat. Junctional Jub forms a complex with the Hippo pathway kinase Warts to downregulate the Hippo pathway and promote growth through activation of Yorkie (Rauskolb et al., 2014; Alégot et al., 2019; Sarpal et al., 2019). This mechanism allows tissue tension to regulate growth and is the only example to date demonstrating a function for $\alpha$ catenin $\mathrm{M}$ region mechanosensing in a whole organism. In particular, how $\mathrm{M}$ region mechanosensing contributes to cell adhesion in tissue morphogenesis remains an open question.

110 The situation is further complicated by the lack of evidence for Vinc as an important player in cell adhesion in vivo. Several tissue culture studies have suggested that the tension-dependent binding of Vinc to the unfurled M1 domain makes an important contribution to adhesion mediated by the CCC (Yonemura et al., 2010; Le Duc et al., 2010; Huveneers et al., 2012; Twiss et al., 2012; Seddiki et al., 2018). In striking contrast, analysis of Vinc mutant animals in C. elegans (Barstead 115 and Waterston, 1991), Drosophila (Alatortsev et al., 1997; Maartens et al., 2016), zebrafish (Han et al., 2017), and mouse (Xu et al., 1998) does not support a significant role of Vinc in cadherinbased cell adhesion. Similarly, Jub null mutants in the mouse, zebrafish and Drosophila complete embryogenesis with only subtle defects (Pratt et al., 2005; Witzel et al., 2012; Razzell et al., 2018) consistent with a minor role in cell adhesion, at best.

The only $\alpha$-catenin $\mathrm{M}$ region binding partner with substantive adhesion defects, although weaker than those resulting from the loss of $\alpha$-catenin, is Afadin/Cno. M3 contains a minimal Afadin binding site, with the exposed M2 domain also contributing to this interaction (Pokutta et al., 2002; Sakakibara et al., 2020). In Drosophila, cno maternal zygotic mutants cause defects in mesoderm 125 invagination (Sawyer et al., 2009) and tears in the ectodermal epithelium as it undergoes convergent extension (Sawyer et al., 2009; Yu and Zallen, 2020). cno zygotic mutants have dorsal closure defects (Jürgens et al., 1984; Takahashi et al., 1998; Boettner et al., 2003; Choi et al., 2011) 
similar to some $\alpha$-Cat zygotic mutants (Sarpal et al., 2012; Jurado et al., 2016). In mouse afadin mutants, strong defects arise after implantation where the neural fold becomes a mass of 130 disorganized cells, embryos remain flat and short and lethality ensues (Ikeda et al., 1999; Zhadanov et al., 1999). Actomyosin cables in cells of the extending germband of Drosophila cno mutants are more diffuse and detached from the membrane, making unproductive contractions (Sawyer et al., 2011). In cell culture, a mutant $\alpha$-catenin isoform with an open conformation of the $M$ region showed increased Afadin recruitment (Matsuzawa et al., 2018), and the $\alpha$-catenin binding site on 135 Afadin is required for its mechanosensitive enrichment (Sakakibara et al., 2020). We wondered therefore whether Cno is acting as the effector of the $\mathrm{M}$ region to support adhesion in Drosophila. The role of $\alpha$-catenin $M$ region domains in Afadin/Cno recruitment and function in adhesion has not been explored in an animal model.

140 The comparatively mild to moderate defects produced by the loss of Vinc, Jub, and Afadin/Cno led us to question (i) whether, and if so, how the $\alpha$-Cat $M$ region contributes to adhesion and embryonic morphogenesis via recruitment of these binding partners in response to force, and (ii) whether there is redundancy between $\alpha$-Cat binding partners which would explain the moderate to subtle phenotypes that result from the loss of Vinc, Jub, and Cno compared to the striking loss 145 of epithelial integrity that is caused by the loss of $\alpha$-Cat. We found that the $\mathrm{M}$ region is required for cell adhesion during early Drosophila embryogenesis, in particular the M2 domain, at contacts that experience higher tension. Our data suggest three distinct tension states read by $\alpha$-Cat mechanosensing that cause the differential recruitment of Vinc, Jub, and Cno to enhance adhesion. Surprisingly, ectopic exposure of the M1 domain was more deleterious than removal the M region entirely. This effect is not due to the recruitment of Vinc, but likely to the M1-dependent regulation of Jub recruitment to AJs. Our findings also support the conclusion that Cno promotes cell adhesion in a parallel pathway to the CCC except at tricellular junctions (TCJs) where the mechanosensitive enrichment of Cno (Yu and Zallen, 2020) depends on the M2 and M3 domains of $\alpha$-Cat. Our work provides evidence of a robust network of cooperative and redundant mechanosensitive interactions at AJs that support tissue morphogenesis. 


\section{Results}

\section{The $M$ region of $\alpha$-Cat is required for epithelial integrity during embryonic morphogenesis.}

We have previously analyzed the ability of mutant $\alpha$-Cat proteins to substitute for endogenous $\alpha$ Cat in several different Drosophila tissues including the embryonic head epidermis, the follicular 160 epithelium of the ovary, and the epithelium of the wing imaginal disc (Sarpal et al., 2012; Desai et al., 2013; Escobar et al., 2015; Ishiyama et al., 2018; Sarpal et al., 2019). Expression of an $\alpha$-Cat construct that lacks the entire $M$ region $(\alpha-C a t-\Delta M)$ could fully support epithelial development of the wing discs, which developed into a normal adult wing (Sarpal et al., 2019). Further analysis of $\alpha$-Cat- $\Delta \mathrm{M}$ showed that it provided a strong rescue of $\alpha$-Cat mutant cells of the follicular epithelium

165 (Fig. S1), supported development of the head epidermis and prevented embryonic lethality of most zygotic $\alpha$-Cat null mutant animals (Sarpal et al., 2019). In contrast to the widely considered model that $M$ region-based mechanosensing enhances adhesion in response to mechanical force (Leckband and De Rooij, 2014; Mège and Ishiyama, 2017; Angulo-Urarte et al., 2020), our in vivo data at this point assigned only a minor or no essential role to the $\mathrm{M}$ region in cell adhesion and

170 epithelial development. We therefore wondered whether $\mathrm{M}$ region function is most relevant during developmental periods of vigorous morphogenesis when cell contacts experience high levels of force. Drosophila gastrulation represents a period of development that condenses several largescale morphogenetic movements into a short $\sim 2$ hour time window. These movements entail the invagination of the mesoderm and endoderm (Martin, 2020), cell intercalations that drive 175 germband extension (Paré and Zallen, 2020), the ingression of neural stem cells (Simões et al., 2017; An et al., 2017), and three rounds of cell division with a 40 minute cell cycle time (Foe, 1989).

To assess $M$ region function in early embryos, we utilized a setup in which the crucial maternal 180 contribution to $\alpha$-Cat expression (Sarpal et al., 2012) is removed through RNA interference (Fig. S2A,B) and constructs are expressed that are resistant to the shRNA used (Sarpal et al., 2019). Our constructs are recruited to AJs (Fig. S2C) and are expressed at close to normal levels (Desai et al., 2013; Sarpal et al., 2019). Resistant constructs contain a mutated shRNA target site that preserves the amino acid sequence located in the M2 domain (denoted by ' $R$ ' ; e.g. $\alpha$-CatR or $\alpha-C a t R-\Delta M 1$, 185 with mutant $\alpha$-Cat isoforms collectively referred to as $\alpha$-CatX) or carry a deletion of M2 (Fig. 1C). Maternally driven $\alpha$-Cat-RNAi caused a dramatic phenotype similar to that previously reported for 
the loss of Arm (Cox et al., 1996) or Ecad (Tepass et al., 1996), leading to a terminal phenotype with a highly fragmented epidermal/cuticle layer (Fig. 1D). We quantified the cuticle defects, which are indicative of adhesion defects, by counting the number of intact abdominal denticle belts. $\alpha$-Cat-RNAi embryos had 0 intact denticle belts. $\alpha$-Cat-RNAi embryos expressing $\alpha$-CatR had all 8 denticle belts restored in $>90 \%$ of animals. In contrast, $\alpha$-Cat-RNAi $\alpha-C a t-\triangle M$ animals showed partial restoration of epidermal integrity compared to $\alpha$-Cat-RNAi, but virtually all animals had denticle belt defects with more than half of embryos producing no denticle belts (Fig. 1C,D). These results demonstrate a substantive requirement of the $\mathrm{M}$ region in maintaining epithelial integrity in the Drosophila embryo.

To investigate the function of the $\mathrm{M}$ region in early morphogenesis, live embryos were observed during gastrulation with a focus on mesoderm invagination and ectodermal integrity during germband extension. Mesoderm invagination failed in $\alpha$-Cat-RNAi embryos (Fig. 2A, Fig. S2B).

200 As previously described (Martin et al., 2010), at the onset of mesoderm invagination actomyosin contraction overpowers residual adhesion in $\alpha$-Cat-RNAi embryos. Cells round up and come apart from each other, with plasma membrane tethers formed between few remaining AJ puncta (Fig. S2D). $\alpha$-Cat-RNAi $\alpha$-CatR embryos showed normal mesoderm invagination. In contrast, $\alpha$-Cat$R N A$ i $\alpha$-CatR- $\triangle M$ embryos displayed a range of defects that were classified according to the degree 205 of successful invagination. $\sim 40 \%$ of embryos showed normal mesoderm invagination where the ventral midline sealed along the entire anterior-posterior axis, whereas the remaining $\sim 60 \%$ of embryos showed either a partial closure or a complete failure to close the ventral midline (Fig. 2A).

210 As the germband starts to extend, ectodermal cells in $\alpha$-Cat-RNAi embryos lose adhesion and large gaps appear between the apical domain of cells (Fig. 2B). Depletion of the maternal contribution of $\alpha$-Cat eventually leads to complete cell dissociation and epithelial collapse (Cavey et al., 2008; Martin et al., 2010; Rauzi et al., 2010; Fernandez-Gonzalez and Zallen, 2011; Wang et al., 2013; Levayer and Lecuit, 2013a; Eritano et al., 2020). Similar to the mesoderm, plasma membrane 215 tethers are seen connecting cells across gaps (Fig. S2E) (Fernandez-Gonzalez and Zallen, 2011). $\alpha$-Cat-RNAi $\alpha$-CatR embryos displayed normal cell contacts in the ectoderm, whereas $\alpha$-Cat-RNAi $\alpha$-CatR- $\Delta M$ embryos showed gaps in the epithelium (Fig. 2B,C). Neighboring apical domains 
became separated, leading to epithelial gaps or tears that extend predominantly along the dorsalventral (DV) axis (Fig. 2D). Membrane tethers can be observed spanning these gaps (Fig. 2B).

220 These defects were associated with a marked reduction in germband extension compared to $\alpha$-Cat$R N A i \alpha$-CatR controls (Fig. 2E). Thus, the M region makes an essential contribution to maintaining adhesion during mesoderm invagination and germband extension and its loss compromises these processes.

225 Exposure of the M1 domain is more deleterious to development than removal of the whole M region.

We next assessed the performance of $\alpha$-Cat constructs that lacked individual $\mathrm{M}$ region domains $(\alpha-C a t R-\Delta M 1, \alpha-C a t-\Delta M 2, \alpha-C a t R-\Delta M 3)$ or both M2 and M3 ( $\alpha$-Cat- $\Delta$ M23). $\alpha$-CatR- $\Delta$ M1 behaved like full-length $\alpha$-CatR when we examined the cuticle (Figs. 1C and S2F), mesoderm 230 invagination (Fig. 2A), the ectodermal epithelium (Fig. 2B-D), and germband extension (Fig. 2E), suggesting that the M1 domain makes no essential contribution to cell adhesion in these tissues. Surprisingly, expression of $\alpha$-Cat- $\triangle \mathrm{M} 23$ was much less capable of ameliorating the $\alpha-C a t-R N A i$ phenotype than $\alpha$-Cat- $\Delta$ M. $\alpha$-Cat-RNAi $\alpha$-Cat- $\Delta M 23$ embryos showed little improvement of the cuticle defects seen with $\alpha$-Cat-RNAi (Figs. 1C and S2F) and failed to rescue mesoderm

235 invagination (Fig. 2A). Most embryos displayed prominent de-adhesion defects in the ectoderm, though these are not as severe as with $\alpha-C a t-R N A i$ alone (Fig. 2B-D), and germband extension was substantially reduced (Fig 2E). Rescue by $\alpha$-Cat- $\Delta \mathrm{M} 2$ showed minor improvements when compared to $\alpha$-Cat- $\Delta \mathrm{M} 23$, whereas expression of $\alpha$-CatR- $\Delta \mathrm{M} 3$ showed a much better rescue than $\alpha$-Cat- $\Delta \mathrm{M} 2$ (Figs $1 \mathrm{C}, 2$, and $\mathrm{S} 2 \mathrm{~F}$ ). This suggests that M2 is the most important domain within the

240 M region for supporting cell adhesion, with M3 making a minor contribution. Notably, the poor rescue activity seen with $\alpha$-Cat- $\Delta \mathrm{M} 23$ compared to $\alpha$-CatR- $\Delta \mathrm{M}$ suggests that the unfurled M1 domain, which is retained in $\alpha$-Cat- $\Delta \mathrm{M} 23$, negatively regulates cell adhesion.

\section{The $M$ region contributes to cell adhesion at medium and high tension cell contact sites}

245 The ectoderm during germband extension displays an asymmetric distribution of non-muscle myosin II, characterized by an enrichment of myosin at vertical cell edges (oriented along the DV axis) and even more so at tricellular junctions (TCJs) (Bertet et al., 2004; Zallen and Wieschaus, 2004; Fernandez-Gonzalez et al., 2009; Tetley et al., 2016; Vanderleest et al., 2018). For the 
purpose of our discussion and reflecting the distribution of myosin, we distinguish between lowtension horizontal bicellular junctions (BCJs), medium-tension vertical BCJs, and high-tension TCJs (vertices). Contractions of myosin at vertical edges either in multicellular rosettes or at bicellular contacts are important drivers of cell intercalation required for germband extension (Zallen and Wieschaus, 2004; Blankenship et al., 2006; Zallen and Blankenship, 2008; FernandezGonzalez et al., 2009; Tetley et al., 2016; Vanderleest et al., 2018) and neuroblast ingression

255 (Simões et al., 2017). We noticed that gaps or tears in the epithelium of embryos expressing M region deletions were commonly found at the centers of rosettes or at vertices, and were elongated along the DV axis (Fig. 2B,D). These findings further suggest that the M region and its individual domains, except for M1, strengthen adhesion and that medium and high tension cell contact sites are more susceptible to loss of adhesion than other cell contacts.

To further characterize these adhesion defects, we analyzed the actomyosin cytoskeleton using the endogenously YFP-tagged myosin heavy chain. The expression of any M region deletion in an $\alpha$ Cat-RNAi background led to increased total myosin signal (Fig. $3 \mathrm{~A}$ and E), while the enrichment of myosin to vertical edges was consistently observed (Fig. 3A). As myosin is recruited by tension 265 (Fernandez-Gonzalez et al., 2009) this suggests that the asymmetric distribution of tension in the ectoderm is not strongly affected in embryos expressing $M$ region deletions. Although membrane association of myosin was preserved even with a poor rescue of adhesion as in $\alpha$-Cat-RNAi $\alpha$-Cat$\triangle M 23$ embryos, separation of apical domains along the DV axis led to the apparent splitting of supracellular myosin cables (Fig. 3B). In embryos with stronger defects, as gaps open between ectodermal cells, myosin accumulates in large puncta at the free apical cell edges (Fig. 3B).

Gaps in the ectoderm of $\alpha$-Cat-RNAi $\alpha$-CatX embryos could also be visualized by an enhanced signal of the active Rhol probe Ani-RBD. Activation of Rho1 causes the phosphorylation of myosin regulatory light chain by Rho kinase, and hence myosin contraction, with enrichments of 275 activated Rho1 seen at sites of high actomyosin contractility in the germband (Munjal et al., 2015; Martino et al., 2018). Loss of apical cell contact was accompanied by the formation of large puncta of Ani-RBD (Fig. 3C,D,F). These observations are reminiscent of the accumulation of active Rho1 and myosin at wound margins (Abreu-Blanco et al., 2014; Rothenberg and Fernandez-Gonzalez, 2019), and Rho flares which repair breaches in the epithelial barrier during Xenopus development 
280 (Stephenson et al., 2019). The appearance of membrane tethers (Fig. 2B) and the accumulation of active Rhol and myosin (Fig. 3) confirmed the loss of cell-cell contacts within the germband, particularly at medium and high tension cell edges or vertices, for embryos expressing deletions that remove the M2 domain (Figs. 2B,C and $3 \mathrm{C}$ ). Moreover, $\alpha-C a t R-\Delta M 3$ and $\alpha-C a t-\Delta M 23$ expressing embryos showed comparatively lower levels than $\alpha$-CatR in both cortical Ani-RBD signal and the proportion of junctional myosin (Fig. 3E,F). In contrast, a higher fraction of junctional myosin was seen when M1 was deleted (Fig. 3E). Together, these findings suggest that the $\mathrm{M}$ region contributes to the regulation of junctional actomyosin.

\section{M2/3 and M1 play opposing roles in regulating AJs}

290 To further characterize the impact of the $\mathrm{M}$ region on cell adhesion we examined the junctional distributions of Ecad and the $\alpha$-Cat binding partners Vinc and Jub using fluorescently-tagged proteins under the control of their endogenous promoters (Huang et al., 2009; Sabino et al., 2011; Kale et al., 2018). In $\alpha$-Cat-RNAi $\alpha$-CatX embryos, the levels of Ecad correlated with the strength of adhesion defects observed (Fig. 4A,C; for a summary of results see Fig. 4D), with lower levels

295 of Ecad associated with more severe defects, as expected. Removal of M2 or M3 caused a significant reduction of cortical Ecad, suggesting that these domains predominantly contribute to the stability of CCC proteins at the junction (Fig 4A,C,D). This concurs with a reduction of junctional Arm in wing disc cells lacking M2 or M3 (Sarpal et al., 2019). In contrast, the M1 domain has an inhibitory effect on Ecad. $\alpha$-Cat-RNAi $\alpha$-CatR- $\triangle M 1$ embryos showed elevated

300 cortical enrichment of Ecad (Fig. 4A,C,D), consistent with an increase in junctional Arm in $\alpha$ CatR- $\Delta$ M1 expressing wing disc epithelium (Sarpal et al., 2019; Alégot et al., 2019). An increase in Ecad levels as a result of the absence of M1 was also seen when comparing $\alpha$-Cat- $\Delta \mathrm{M} 23$ and $\alpha$ Cat- $\Delta \mathrm{M}$ embryos, and $\alpha-C a t-\Delta \mathrm{M}$ appears to be better maintained at the membrane and is less punctate than $\alpha$-Cat- $\Delta \mathrm{M} 23$ (Figs. 4 and S2A). We conclude that the M region regulates junctional 305 stability with M1 and M2/3 having opposing effects. Whereas M2/3 stabilizes the junction, M1 limits junctional Ecad.

\section{$\mathrm{M} 2$ and M3 negatively regulate $\mathrm{M1}$ function}

M1 is required for all detectable junctional Vinc signal in gastrulating embryos (Fig. 4A,C,D). As removal of M2 and M3 also reduces Ecad, we wanted to know whether the reduction in Vinc in 
these conditions is explained by a reduction of the CCC. We therefore calculated the ratio of cortical Vinc to Ecad per cell (expressed within the same embryo). We found that the M2 and M3 domains contribute to Vinc junctional levels even when normalized to the amount of remaining Ecad (Fig. 4A,C,D). Removal of both M2 and M3 together significantly increases the Vinc/Ecad 315 ratio compared to $\alpha$-CatR control (Fig. 4A,C,D). Together, this suggests that both M2 and M3 individually inhibit M1, but also each other, such that removing M2 and M3 together relieves all inhibition of Vinc recruitment (Fig. 4E). Removing M2 alone leaves M3 free to inhibit the M1Vinc interaction and vice versa. This is in agreement with work using cells in culture identifying a masking effect between domains (Yonemura et al., 2010; Matsuzawa et al., 2018; Sakakibara et al., 2020), and provides evidence within an animal model to support the existence of autoinhibition among $\mathrm{M}$ region domains.

\section{M1 inhibits the $\alpha$-Cat-Jub interaction during germband extension}

In wing disc epithelial cells expressing M2 or M3 deletions instead of endogenous $\alpha$-Cat, Jub is

325 reduced likely due to an overall reduction in CCC levels. However, Jub levels are strikingly increased in cells lacking M1 (Sarpal et al., 2019; Alégot et al., 2019). We observed a similar pattern in the early embryo. Embryos where M1 is removed showed a dramatic increase in Jub at the junctions (Fig. 4B,C,D). In contrast, Jub levels are reduced in $\alpha$-Cat-RNAi embryos expressing $\alpha$-Cat- $\Delta \mathrm{M} 2, \alpha$-CatR- $\Delta \mathrm{M} 3$, or $\alpha$-Cat- $\Delta \mathrm{M} 23$, suggesting that the M2 and M3 domains support

330 junctional Jub. Despite lacking M2 and M3, embryos expressing $\alpha$-Cat $\Delta \mathrm{M}$ experience an enrichment of Jub (Fig. 4B,C,D). Thus, as shown for the wing disc epithelium (Sarpal et al., 2019; Alégot et al., 2019), M1 also acts as an inhibitor of junctional Jub recruitment in the embryonic ectoderm. Ectopic exposure of the M1 domain in $\alpha$-Cat-RNAi $\alpha$-Cat- $\Delta M 23$ embryos produces the largest reduction in Ecad, Jub (Fig. 4), and the strongest adhesion defects (Figs. 1C, 2, S2F). As

335 junctional Jub has a positive role in cell adhesion in the ectoderm (Razzell et al., 2018), we conclude that M1 is a negative regulator of cell adhesion, a role normally limited by the functions of M2 and M3, an inhibition resolved by the force-dependent unfurling of the M region.

\section{The M2 domain is required for the mechanosensitive enrichment of Jub at high tension edges}

340 We also found that manipulation of the $M$ region significantly affected the mechanosensitive recruitment of Jub. Jub is recruited to the membrane in response to cytoskeletal tension (Rauskolb 
et al., 2019; Razzell et al., 2018; Alégot et al., 2019), In wing disc epithelium, removal of the M1 domain causes Jub to become hyper-recruited to junctions without an increase in tissue tension (Sarpal et al., 2019; Alégot et al., 2019). How this occurs is unclear as the Jub-binding site is not within the $\alpha$-Cat $\mathrm{M}$ region, but in the N2 domain (Marie et al., 2003; Alégot et al., 2019; Sarpal et al., 2019). A requirement for the $\alpha$-Cat N-terminal domain for the junctional recruitment of Jub was confirmed in the embryo, as rescue with a construct which lacks this domain (DEcad $\Delta \beta:: \alpha-$ Cat-ABD) depleted Jub levels to the same degree as $\alpha$-Cat knockdown (Fig. 5A-C). During germband extension, where vertical edges are under higher tension than horizontal contacts, this leads to the planar polarized enrichment of Jub to vertical edges (Razzell et al., 2018). This provided us with a system in which we could test Jub response to tension. We determined the planar polarity of Jub in $\alpha$-Cat-RNAi $\alpha$-CatX embryos by measuring the average fluorescent intensity of Jub along vertical edges divided by that of horizontal edges within the same embryo. This analysis revealed that the M2 domain is in fact required for the specific enrichment of Jub to vertical cell contacts (Fig. 5D). Expression of $\alpha$-Cat- $\Delta M, \alpha-C a t-\Delta M 23$, and $\alpha$-Cat- $\Delta M 2$ failed to rescue the enrichment of Jub to edges approaching $90^{\circ}$ (where the anterior-posterior axis $=0^{\circ}$ ), and in some instances a reversal of Jub planar polarity was seen with higher Jub levels at horizontal versus vertical edges (Figs. 5D and S3A).

360 The planar polarity of Jub in $\alpha-C a t-R N A i \alpha-C a t-\triangle M 1$ embryos is also significantly reduced, but in this case this is due to a constitutive enrichment of Jub to all cell edges, regardless of the state of tension (Figs. 5A-D and S3A). Similarly, in wing disc epithelium, loss of M1 desensitizes junctional Jub recruitment to a reduction of myosin activity (Alégot et al., 2019). The planar polarity of Jub thus requires M2 for the mechanosensitive recruitment to higher tension edges,

365 while M1 limits Jub at lower tension edges. Although Jub is required for normal germband extension, its planar polarity is not essential for adhesion or germband extension (Razzell et al., 2018). The M2 domain may therefore function by supporting the necessary amount of Jub at higher tension edges, and hence support Ecad membrane stability against enhanced actomyosin contractility. As junctional Jub is more enriched in $\alpha$-Cat- $\Delta \mathrm{M}$ than $\alpha$-Cat- $\Delta \mathrm{M} 23$ embryos, we

370 wondered whether the lack of M1-mediated inhibition of Jub helps to explain the difference in rescue of cell adhesion by these two constructs. Indeed, depletion of Jub by RNAi worsened epithelial integrity of $\alpha$-Cat-RNAi $\alpha$-Cat- $\triangle M$ embryos (Fig. 5E). These results suggest that Jub and 
the M2 domain cooperate with each other to support AJs under morphogenetic stress, and underline a requirement for the $\mathrm{M}$ region for the mechanosensitive recruitment of Jub to edges 375 under higher tension.

\section{The $M$ region reinforces Ecad against enhanced actomyosin contraction}

In contrast to the enrichment of the $\alpha$-Cat binding partners Jub, Vinc and Cno at vertical, high tension edges during germband extension (Blankenship et al., 2006; Sawyer et al., 2011; Kale et al., 2018; Razzell et al., 2018), AJ components such as Baz, Arm, $\alpha$-Cat and Ecad become planar polarized and enriched at horizontal edges (Paré and Zallen, 2020). Within an Ecad::GFP, GAP43::mCherry background, embryos expressing $\alpha$-Cat-RNAi $\alpha-C a t-\Delta M$ and $\alpha$-Cat-RNAi $\alpha$ Cat- $\triangle M 23$ showed significantly enhanced planar polarized localization of Ecad. Ecad was reduced specifically from the actomyosin-enriched vertical edges compared to $\alpha$-CatR controls (Fig. 6A), suggesting that the M2 and M3 domains support Ecad at high-tension edges. Interestingly, within an Ecad::GFP, Vinc::mCherry background, a reversal of Ecad planar polarity was seen in $\alpha$-CatRNAi $\alpha$-Cat- $\triangle M 23$ embryos (in which Vinc is ectopically enriched at AJs), while there was no change to Ecad planar polarity in embryos rescued by $\alpha$-Cat- $\Delta \mathrm{M}$ or $\alpha$-CatR- $\Delta \mathrm{M} 1$ (which cannot bind Vinc) (Fig. 6A). Expression of Vinc::mCherry to assess Vinc distribution provided an additional genomic copy of Vinc (Kale et al., 2018), representing an over-expression condition. This additional copy of Vinc also improves adhesion in the lateral ectoderm of $\alpha$-Cat-RNAi $\alpha$-Cat$\triangle M 23$ embryos compared to controls without Vinc::mCherry (Fig. 6B). As another method to enhance Vinc activity, we expressed the constitutively active Vinc-CO mutation (Maartens et al., 2016). This caused a strong enrichment of Ecad (Fig. 6C), and a reversal of Ecad planar polarity such that Ecad is enriched at vertical edges (Fig. 6D). These results suggest that the M1-Vinc interaction can reinforce Ecad recruitment or stability in response to actomyosin contraction and, consequently, cell adhesion during Drosophila gastrulation. However, as the M1 domain is dispensable for adhesion and embryonic development, it is unlikely that the recruitment of Vinc is the primary mode of junctional reinforcement through the $\mathrm{M}$ region in wildtype. Instead, our data suggests that M1 functions as an inhibitor of AJs. 


\section{The M1 domain is required for the normal distribution of junctional Ecad through recruitment of Vinc}

405 Since M1 has an inhibitory effect on Ecad and Jub, and over-exposure of M1 both enriches Vinc and causes more severe defects than removal of the whole M region, we wondered whether Vinc has an inhibitory effect on Ecad or Jub, and thus adhesion. A Vinc null mutant allele was incorporated into the rescue set-up to see if this would ameliorate epithelial integrity defects in $\alpha$ Cat-RNAi $\alpha$-Cat-AM23 embryos. However, removal of Vinc in fact worsened the defects in $\alpha$-Cat-

$410 \Delta \mathrm{M} 23$ embryos, suggesting that Vinc cooperates with the M2 and M3 domains to support adhesion (Fig 6E). Furthermore, we confirmed that Vinc null mutants have no discernable epithelial defects during gastrulation, as previously reported (Alatortsev et al., 1997; Maartens et al., 2016), but found subtly yet significantly reduced $\alpha$-Cat and Jub levels (Fig. 6F,H). Although M1 is similarly dispensable for embryonic development, abnormally large clusters of Ecad, Jub, and $\alpha$-Cat- $\Delta \mathrm{M} 1$

415 were seen in $\alpha$-Cat-RNAi $\alpha$-Cat- $\Delta M 1$ embryos along the junctions compared to $\alpha$-CatR expressing controls (Figs. 5A, 6G, S4A). We used the normalized standard deviation of Ecad to estimate the fragmentation of junctional signal, and this was significantly increased in the absence of M1 (Fig. 6G). Likewise, junctional $\alpha$-Cat signal is more fragmented in Vinc embryos (Fig. 6H). M1 recruitment of Vinc therefore does support a more uniform distribution of the CCC at AJs, but the

420 impact of its loss has no apparent phenotypic consequences. Our observations suggest that the inhibitory effect the M1 domain has on adhesion is not due to Vinc recruitment, and that the M1 domain plays a dual inhibitory and supportive role at AJs.

\section{Cno requires M2/M3 for its mechanosensitive enrichment to TCJs, but is cortically recruited}

\section{5 independently of the $\alpha$-Cat $M$ region}

Loss of either Vinc or Jub alone did not produce significant adhesion defects in Drosophila embryos (Maartens et al., 2016; Razzell et al., 2018), and also Jub knockdown in a Vinc mutant background did not enhance embryonic lethality. However, loss of Vinc or Jub enhanced epithelial defects in $\mathrm{M}$ region deletion construct expressing embryos (Figs. 5E and 6E), raising the possibility

430 that they work in parallel with an additional interaction partner of the $\mathrm{M}$ region, contributing to its mechanosensory output. As a known binding partner of the mammalian $\alpha$-catenin M2/M3 domains (Pokutta et al., 2002; Sakakibara et al., 2020), we examined therefore the Drosophila Afadin 
ortholog Cno to assess if force-dependent recruitment of Cno by the M region could help explain $\mathrm{M}$ region function in cell adhesion.

Although a masking effect of M1 on the M3-Afadin interaction was reported in MDCK cells (Sakakibara et al., 2020), in the Drosophila embryo we found that removal of either M3 or M1 led to an increase in junctional Cno (Fig. 7A,B). In fact, junctional Cno increased modestly with the expression of all $\mathrm{M}$ region deletions except for $\alpha-C a t-\Delta \mathrm{M} 23$, which showed a reduction but not a loss of Canoe at AJs (Fig. 7A,B). By stage 8, Cno is significantly reduced by the knockdown of $\alpha$-Cat alone (Fig. 7A,B), when few cell junctions remain. Since Cno levels are increased in $\alpha$-Cat$\Delta \mathrm{M}$ expressing embryos, the reduction of Cno in $\alpha$-Cat-RNAi $\alpha-C a t-\Delta M 23$ embryos may be a consequence of the strong adhesion defects in this condition. Although the planar polarity of Cno in $\alpha$-Cat-RNAi $\alpha$-CatR- $\triangle M 3$ embryos was reduced, Cno planar polarity was unaffected in $\alpha$-Cat$R N A i \alpha$-Cat- $\Delta M$ (Fig. S3). This did not allow us to conclude that M3 is required for the enrichment of Cno at vertical edges.

Cno is normally elevated both at vertical BCJs during gastrulation and also at TCJs (Sawyer et al., 2009; 2011). The enrichment of Cno at TCJs is responsive to cytoskeletal tension (Yu and Zallen, 450 2020). We therefore assessed Cno levels at vertices as a TCJ/BCJ ratio within the same embryo. Although the minimal Afadin binding site of $\alpha$ E-catenin is located within M3 (Pokutta et al., 2002; Sakakibara et al., 2020), removing M3 alone had no effect on the TCJ enrichment of Cno (Fig 7A,D). However, the enrichment of Cno at vertices was significantly reduced in embryos lacking M2, and this is worsened when M3 is also removed (Fig. 7A,D). These results suggest that M2 455 and M3 can interact with Cno independently and that M3 supports M2 in the mechanosensitive recruitment of Cno to TCJs.

The pools of Cno at BCJs versus TCJs thus appeared to have different requirements for the $\mathrm{M}$ region. Interestingly, $\mathrm{Cno}$ at $\mathrm{BCJ}$ s does not show a response to experimental changes in tension 460 (Yu and Zallen, 2020). Measurements of cortical Cno represent predominantly the bicellular pool, which does not require the $\mathrm{M}$ region (Fig. 7B). Loss of the $\mathrm{M}$ region also does not abolish Cno localization to TCJs (Fig. 7C,D). Cno must therefore have alternate pathways to associate with the plasma membrane. During cellularization, Cno recruitment is unaffected by the loss of Arm, and 
instead requires the small GTPase Rap1 (Sawyer et al., 2009). Echinoid (Ed) is thought to cooperate with Ecad to support Cno recruitment (Wei et al., 2005; Sawyer et al., 2009). However, even in a naturally occurring Ed deficient tissue, the amnioserosa (Lin et al., 2017), junctional recruitment of Cno was not depleted in $\alpha$-Cat-RNAi $\alpha$-CatR- $\Delta M 3$ or $\alpha$-Cat-RNAi $\alpha-C a t R-\Delta M$ embryos (Fig. S4). Deletion of M1 exposes the Cno binding site, but bicellular $\alpha$-Cat- $\Delta \mathrm{M} 1$ signal does not colocalize better with Cno than $\alpha$-CatR control when expressed in $\alpha$-Cat-RNAi embryos

470 (Fig. S4), further supporting the conclusion that Cno can localize to BCJs independently of the M region. Finally, $\alpha$-Cat constructs were also consistently located more basally than Cno at stage 8 (Fig. S4). Taken together, these findings suggest that Cno is recruited to the membrane independently of the $\alpha$-Cat $\mathrm{M}$ region, but that the mechanosensitive recruitment of a pool of Cno to TCJs requires an interaction with the $\alpha$-Cat M2 and M3 domains.

\section{The $\alpha$-Cat $M$ region acts in parallel to Cno to support cell adhesion}

If $\mathrm{M}$ region mechanosensing supports adhesion by recruiting $\mathrm{Cno}$ as a downstream effector, then compromising Cno within an embryo lacking the M2 and/or M3 domains should not worsen adhesion defects. However, Cno knockdown enhanced the epithelial defects of all $\alpha$-Cat deletion 480 constructs (Fig. 8A). Furthermore, overexpression of Cno improves epithelial integrity in embryos expressing $\alpha$-Cat-RNAi alone (Fig. 8B), and constructs lacking the M2 and M3 domain (Fig. 8C). In particular, overexpression of Cno significantly ameliorated the loss of ventral epithelium in $\alpha$ Cat-RNAi $\alpha$-Cat- $\triangle M 23$ and $\alpha$-Cat- $\Delta M$ embryos, which are predicted to abolish Cno binding, as well as $\alpha$-Cat- $\Delta \mathrm{M} 2$ (Fig. $8 \mathrm{C}, \mathrm{D})$. Together with the $\mathrm{M}$ region-independent association of Cno with

485 AJs, these observations suggest that Cno supports adhesion in an $\alpha$-Cat-independent parallel pathway. In further support of this conclusion we found that knockdown of the Rap1 GTPase, which contributes to Cno membrane recruitment and activation (Sawyer et al., 2011; Bonello et al., 2018; Perez-Vale et al., 2021), enhances the defects in $\alpha$-Cat-RNAi $\alpha$-CatR- $\Delta M$ embryos (Fig. 8E). These results suggest that Rap1 interaction with Cno shares some parallel function with the 490 M region to support adhesion. Although these results do not rule out a role for $\mathrm{M}$ region-dependent Cno recruitment, it argues against Cno acting solely as an effector of $\alpha$-Cat mechanosensing. Instead, Cno appears to have a redundant role to the M region, and to the M1 domain in particular as knockdown of Cno enhances loss of junctional Ecad and cell adhesion defects in $\alpha-C a t R-\Delta M 1$ embryos compared to $\alpha$-CatR controls (Fig. 8A,D). 


\section{a-Cat binding partners and $\mathrm{Cno}$ form a cooperative network to support adhesion}

We wondered whether the recruitment of Vinc by M1 is redundant with Cno function. Epithelial integrity was significantly worsened when Vinc is removed from a cno-RNAi background, consistent with functional redundancy between Cno and the M1-Vinc interaction (Fig. 8F).

500 Functional redundancy between Cno and the M1 domain could explain why M1, and similarly, Vinc are dispensable in the embryo. Furthermore, double knockdown of Jub and Cno significantly enhanced adhesion defects compared to Cno knockdown alone (Fig. 8G). Such findings argue for a model where multiple ways to stabilize Ecad build a robust partially redundant network of interactions to foster AJ stability. Our evidence supports a model where the $\alpha$-Cat $\mathrm{M}$ region itself, and the $\alpha$-Cat binding partners Vinc and Jub cooperate with Cno to promote cell adhesion. 


\section{Discussion}

$\alpha$-catenin is essential for cell adhesion in most animal tissues. $\alpha$-catenin acts as a physical linker between the cadherin/ $\beta$-catenin complex and the actin cytoskeleton (Desai et al., 2013; Buckley et 510 al., 2014), and through its mechanosensory properties has the potential to modify interactions between cadherins and the cytoskeleton in response to external or internal forces (Yonemura et al., 2010; Le Duc et al., 2010; Borghi et al., 2012; Buckley et al., 2014; Ishiyama et al., 2018; Xu et al., 2020). Two mechanosensory regions within $\alpha$-catenin have been identified: the C-terminal actin-binding domain of $\alpha$-catenin increases binding strength to F-actin as force is applied

515 (Buckley et al., 2014; Ishiyama et al., 2018; Xu et al., 2020), and the central M region of $\alpha$-catenin that acts principally by modifying interactions between $\alpha$-catenin and binding partners in response to force (Yonemura et al., 2010; Ishiyama et al., 2013; Thomas et al., 2013; Yao et al., 2014; Li et al., 2015; Maki et al., 2016; 2018; Seddiki et al., 2017; Barrick et al., 2018; Matsuzawa et al., 2018; Terekhova et al., 2019; Sarpal et al., 2019; Alegot et al., 2019). How $\alpha$-catenin mechanosensing cooperates with multiple known $\alpha$-catenin binding partners that can also bind to F-actin to dynamically adjust adhesion during tissue morphogenesis has remained largely unexplored.

We determined that the $\mathrm{M}$ region of Drosophila $\alpha$-Cat plays a key role in maintaining cell adhesion during mesoderm invagination and cell intercalation that drives axis elongation in the early embryo. Taking advantage of the well-described tension landscape in the ectoderm as it undergoes cell intercalation during germband extension (Bertet et al., 2004; Fernandez-Gonzalez et al., 2009; Tetley et al., 2016), we were able to identify three different tension states of $\alpha$-Cat that correspond to the differential recruitment of binding partners (Fig. 9). (i) Moderate amounts of Vinc and Jub

530 are recruited to AJs at low-tension BCJs (=horizontal junctions in the extending germband); (ii) At medium-tension BCJs (=vertical junctions in the extending germband) recruitment of Vinc and Jub is significantly increased; and (iii) at TCJs, which experience the highest levels of tension, Vinc and Jub are further elevated, and Cno is enriched in response to $\alpha$-Cat mechanosensing. Recruitment of Vinc and Jub to AJs critically depends on physical interaction between Vinc and 535 the M1 domain and Jub and the N2 domain. In contrast, Cno localizes at AJs via mechanisms that are independent of $\alpha$-Cat and supports cell adhesion in a parallel pathway. Only the 
mechanosensitive enrichment of Cno at tricellular vertices ( $\mathrm{Yu}$ and Zallen, 2020) requires its interaction with the M2 and M3 domains of $\alpha$-Cat.

540 The $\mathrm{M}$ region of $\alpha$-catenin is stabilized by a dynamic network of salt-bridges (Ishiyama et al., 2013; Li et al., 2015). Single molecule force measurements (Yao et al., 2014; Maki et al., 2016) suggest that the autoinhibited conformation can open when forces above approximately $5 \mathrm{pN}$, the range of force generated by a single myosin motor protein (Finer et al., 1994). A $5 \mathrm{pN}$ force unfurls the M1 domain which becomes accessible to Vinc, an interaction that stabilizes the open (M1 545 unfurled) conformation of $\alpha$-catenin (Yao et al., 2014; Maki et al., 2016). A further conformational change, likely affecting the M2 and M3 domains takes place when a $\sim 10-15 \mathrm{pN}$ force is applied (Yao et al., 2014). Finally, the M1-Vinc interaction is destabilized at forces above $30 \mathrm{pN}$, and Vinc dissociates from $\alpha$-catenin (Yao et al., 2014). Projecting these findings onto our results, we suggest that the difference between horizontal and vertical edges is in the frequency by which $\alpha$-Cat is exposed to low ( $\leqq 5 \mathrm{pN})$ or medium $(5-10 \mathrm{pN})$ forces (Fig. 9 A-C). At vertical edges, medium tension/forces prevail that cause the majority of $\alpha$-Cat molecules to adopt an M1 unfurled conformation which allows both Vinc binding to M1 and Jub recruitment to N2. The unfurled M1 conformation represents a smaller fraction of $\alpha$-Cat molecules at horizontal edges causing a reduced amount of Vinc and Jub recruitment. Finally, forces between $\sim 10$ and $30 \mathrm{pN}$ per individual $555 \alpha$-Cat molecule at TCJs would allow $\alpha$-Cat to engage not only high levels of Vinc and Jub but also interact with Cno to further stabilize AJs (Fig. 9 A-C). A majority of $\alpha$-Cat molecules are unlikely to experience forces above $30 \mathrm{pN}$ as a loss of Vinc from the membrane is not observed even at TCJs.

560 The M1, M2 and M3 domains of $\alpha$-Cat make distinct contributions to cell adhesion. M2 has a major role in supporting surface stability of the CCC and is required for the mechanosensitive AJ enrichment of Jub, which further stabilizes the junction. Together with M3, M2 supports Cno recruitment to TCJs to further reinforce AJs (Fig. 9). Recently, mechanical coupling between M2 and N2 domains has been identified (Terekhova et al., 2019), as well as direct, transient interaction 565 between the disordered N-terminal region of $\beta$-catenin and the $\mathrm{M}$ region (Bush et al., 2019), suggesting that M2 may participate in strengthening interactions at the $\mathrm{N}$-terminal domain with Jub and Arm. In contrast, M1 negatively regulates adhesion by inhibiting Jub recruitment, this 
negative regulation is released by M1 unfurling in response to force. While M1 recruitment of Vinc supports AJs stability, this contribution is subtle and dispensable for adhesion in wild-type.

A noticeable effect of the loss of M1 or Vinc was a somewhat enhanced clustering of the CCC suggesting that the M1-Vinc interaction supports the uniform distribution of the CCC at cell contacts. Genetic analysis suggests that the M1-Vinc interaction is redundant with Cno and that Cno and the $\mathrm{M}$ region act in parallel to support AJs.

575 The M23 and M1 domains appear to have opposing roles in adhesion, similar to their function in the regulation of tissue growth (Sarpal et al., 2019). Whereas M1 has an important role in growth regulation, it is largely dispensable for adhesion, and the opposite is largely true for the M2 and M3 domains. This suggests that $\mathrm{M}$ region mechanosensing acts similarly in cell adhesion and tissue growth and that the tissue-specific consequences are due to differences in the downstream

580 pathways that impact on growth versus adhesion. Our data would also suggest that Vinc does not play a significant role in either function (Sarpal et al., 2019). However, our results imply that Vinc supports adhesion in a subtle and redundant way. This is consistent with a previously reported worsening of the zygotic $\alpha$-Cat mutant phenotype in combination with a Vinc mutation (Jurado et al., 2016). In embryos lacking M2, cortical levels of both Jub and Vinc, as well as Cno enrichment

585 at TCJs, are reduced. Effects on multiple binding partners at once could could explain the strong adhesion defects observed in these embryos. Additional $\alpha$-Cat binding partners such as $\alpha$-Actinin, which also interacts with M1 (Nieset et al., 1997), remain to be investigated in our system, and could contribute to the redundant, multivalent interactions at the CCC-actin interface.

590 Recent analysis of Cno function in the Drosophila ectoderm highlighted the need for multiple, partially redundant interactions between Cno, the plasma membrane, and the cytoskeleton in support of AJ stability during tissue morphogenesis (Manning et al., 2019; Perez-Vale et al., 2021). Thus, both Cno and $\alpha$-Cat stabilize AJs through multiple interactions. It is unclear, however, whether Cno is a mechanosensor or only responds to mechanosensory inputs by $\alpha$-Cat as we show 595 here or by other mechanisms as Cno enrichment at TCJs is also enhanced by phosphorylation of Cno by the Abl non-receptor tyrosine kinase (Yu and Zallen, 2020). Whether phosphorylation of Cno modifies the interactions with $\alpha$-Cat remains to be explored. Cno at TCJs needs to be dynamic 
to enable the normal vertex resolution during the cell rearrangements required for cell intercalation (Yu and Zallen, 2020).

600

Taken together, our work suggests that the $\alpha$-Cat $M$ region acts in cell adhesion to support embryonic morphogenesis through mechanosensitive modulation of interactions with Vinc, Jub, and Cno. The M1-Vinc interaction, dispensable on its own, shows redundancy with Cno function. While Vinc and Jub association with AJs is completely dependent on $\alpha$-Cat mechanosensing, the 605 association of Cno with AJs depend on $\alpha$-Cat mechanosensing only for its enrichment at TCJs. Cno supports adhesion at bicellular contacts in a parallel pathway to $\alpha$-Cat. We suggest that these redundant and cooperative multivalent interactions are the molecular basis of the mechanoresponsive dynamic stabilization of AJs that maintain tissue integrity as the tissue undergoes cell contacts changes. 


\section{Materials and Methods}

\section{Drosophila Genetics}

615 Flies were raised on standard media at $25^{\circ} \mathrm{C}$ for all experiments. For rescue experiments, males carrying mat-GAL4 (P\{mata4-GAL-VP16\}67 (Häcker and Perrimon, 1998) and the $\alpha$-Catenin constructs (inserted at attP2) were crossed to females carrying fluorescently tagged proteins of interest and UAS- $\alpha$-Cat-RNAi (TRiP HMS00317, Transgenic RNAi project [TRiP]). F1 virgin females carrying mat-GAL4, fluorescent protein, and $\alpha$-Cat construct were then crossed to

620 OregonR wild-type males (except jub::GFP males were used for rescue experiments measuring $j u b:: G F P)$ and the progeny of this cross were analyzed. Similarly, for double knockdown analysis, F1 virgin females carrying both mat-GAL4 and transgenes were crossed to OregonR wild-type males and their progeny assessed. In Vinc mutant analysis, a complete genomic deletion of Vinc, $V_{\text {Vinc }}{ }^{102.1}$ was used (Klapholz et al., 2015). See Supplemental Table 1 for details of crosses used for 625 each figure. The following fly lines were employed:

UAS constructs: $\alpha-C a t R, \alpha-C a t R-\Delta M 1, \alpha-C a t-\Delta M($ aka: $\alpha-C a t R-\Delta M), \alpha-C a t R-\Delta M 3$ (Sarpal et al., 2019); $\alpha$-Cat- $\Delta M 2$ (aka: $\alpha C a t \Delta V H 2-N$ ), $\alpha$-Cat- $\Delta M 23$ (aka: $\alpha C a t \Delta V H 2$ ), DE-cad $\Delta \beta:: \alpha$ CatABD (aka: DE-cad $\triangle \beta:: V H 3-C T D$ ); UAS-GFP (Desai et al., 2013); Vinc-CO (Maartens et al 630 2016); UAS-Canoe (aka: CanoeFL::GFP) (Bonello et al., 2018).

RNAi lines: $\alpha$-Cat-RNAi (TRiP HMS00317); jub-RNAi (TRiP HMS00714); canoe-RNAi (TRiP HMS00239 and GL00633); Rap1-RNAi (TRiP HMJ21898); were produced by the Transgenic RNAi Project at Harvard (Zirin et al., 2020). GFP-RNAi (BL41552, Perrimon, N.).

Fluorescently tagged markers: $\alpha$-Cat::YFP ( $\alpha$-CatCPTI002516); Canoe::YFP (cnoCPTI000590); and Zipper::YFP (zipCC01626) are derived from the CPTI protein trap project, Kyoto Stock Centre (Lye et al., 2014; Lowe et al., 2014). Other fluorescently tagged proteins are insertions under the control of the respective endogenous promoter: Ecad::GFP, aka: DEcad::GFP, shg>DEcad::GFP (Huang et al., 2009); Vinc::mCherry (recombined with Ecad::GFP) (Kale et al 2019); jub::GFP (Sabino et al., 2011) Fluorescently tagged proteins ubiquitously expressed in the 
early embryo: Ani-RBD aka:Ubi>Anillin-RBD::GFP (Munjal et al., 2015) and sqh>GAP43::mCherry (Martin et al., 2010).

\section{Preparation of cuticle and quantification of embryonic lethality}

To analyze the terminal cuticle phenotype of embryos, eggs collected overnight at $25^{\circ} \mathrm{C}$ were aged for 2 days or 24 hours if viable, and then washed in water and dechorionated for 5 mins in 2.15\% sodium hypochlorite. After a second wash, embryos were mounted on a slide into a 3:2 mixture of Hoyer's medium and lactic acid and incubated at $85^{\circ} \mathrm{C}$ overnight. To determine the percentage of 650 embryonic lethality, flies were allowed to lay eggs at $25^{\circ} \mathrm{C}$ for an 8 hour period, and between 100 300 eggs were counted and arranged into rows on a new agar plate. This plate was then examined 24 and 48 hours later to count hatched larvae and dead embryos.

\section{Antibody staining}

655 Drosophila embryos were dechorionated in 2.15\% sodium hypochlorite and then either heat-fixed in a salt solution (Miller et al., 1989) (Fig. 8, S4) or fixed for $20 \mathrm{~min}$ in 4\% formaldehyde in a 1:1 PBS:heptane mixture (Fig S2). Primary antibodies used under heat fixation were anti-HA (rat monoclonal, 3F10; 1:500; Roche), anti-Canoe (rabbit 1:1000, a gift from Mark Peifer, University of North Carolina, Chapel Hill, NC, USA), anti-DE-cadherin (rat monoclonal DCAD2 1:25;

660 Developmental Studies Hybridoma Bank). Under heptane fixation: anti-HA (mouse 16B12 1:100, Abcam).

\section{Analysis in follicular epithelium}

A heat-shock inducible MARCM system was employed as previously described (Sarpal et al., 665 2012; Desai et al., 2013). For cell clones produced in the follicular epithelium, the percentage of rescued cells was calculated. The following recombinant lines were used for MARCM analysis: $h s-F l p \quad F R T 40 A ; \quad d a-G a l 4 \quad U A S-m C D 8:: G F P \alpha-C a t^{1} / T M 6 B ;$ tub-Gal80 ubi- $\alpha$-Cat FRT40A; act-Gal4 $\alpha$-CatX $\alpha$-Cat1/TM6b (Sarpal et al., 2012; Desai et al., 2013; Sarpal et al., 2019).

\section{Imaging and signal intensity quantification}


Live imaging was performed on dechorionated embryos mounted in Halocarbon Oil 25 between an oxygen permeable membrane and coverslip, and short time-lapse movies were acquired, similar to other previously described methods (Blankenship et al., 2006). Fluorescent images were 675 acquired using a Leica TCS SP8 scanning confocal microscope on $40 \times$ or $63 \times$ objectives (HC PL APO CS2 with NAs of 1.30 and 1.40, respectively). A Carl Zeiss Axiophot2 microscope using a phase-contrast $20 \times$ lens (NA 0.5) connected to a Canon Rebel XSi camera was used to capture images of embryonic cuticles, and time-lapse movies were captured using this scope and a 10x lens (NA 0.14) to determine rates of germband extension. The number of embryos per genotype is listed on each figure, and in Supplemental Table 1.

Z-stacks were collected using a z-step size of 0.35-0.4 $\mu \mathrm{m}$, starting from above the vitelline membrane and moving well through all visible apical junctional structures for 15-20 steps. Planes containing autofluorescence of the vitelline membrane were removed in post-processing and maximum projections were produced using Fiji (Schindelin et al., 2012). Adobe Illustrator was used to place confocal images and curves were adjusted in Adobe Photoshop only for Vinc::mCherry confocal images and images of cuticle preparations. For images shown using a colormap legend, the 'gem' lookup table was used. The same settings on confocal equipment and image processing were applied for all images within the same experiment.

\section{Analysis of early embryonic phenotypes and germband extension: $\alpha$-Cat-RNAi $\alpha$-CatX} expressing embryos were live imaged during stages 6,7 and 8 and categorization was determined for embryos within a Jub::GFP, Ecad:GFP, GAP43::mCh, or myosin::YFP background (Supplemental Table 1). For quantification of germband extension, the change in position of the proctodeal invagination after 40 minutes was normalized to the total length of the embryo.

Fluorescent Intensity: Analysis of cortical levels of fluorescence was performed using the Matlab script SIESTA (scientific image segmentation and analysis (Fernandez-Gonzalez and Zallen, 2011). For a number of embryos (e), a polyline 3 pixels in width was drawn along the perimeter of each cell (c), and the mean fluorescent intensity along this line, minus the mean fluorescent intensity within the center of the cell, was plotted as cortical fluorescent intensity (FI). Total junctional myosin is given as the sum of the mean fluorescence along the perimeter and mean 
fluorescence within the perimeter for each cell (total FI). Junctional/total myosin fraction is given as the average FI of myosin at the cell perimeter (junctional) out of total myosin (fraction of total FI). For comparison of Ani-RBD at the edge of gaps versus cells not in contact with a gap, the average fluorescent intensity along the perimeter (perimeter FI) was plotted for polylines drawn along cells (intact) versus along the gap (gap).

Planar polarity: Using SIESTA, trajectories 3 pixels in width were drawn along individual cell 710 edges, avoiding overlap or vertices and these edges were grouped into $15^{\circ}$ bins, reflected about the DV axis. For each bin, the cytoplasmic fluorescence of the image was subtracted from the average fluorescent intensity of cell edges. This value was then divided by that of the 0-15 (horizontal) bin. Enrichment to vertical edges is plotted from this calculation for the $75-90^{\circ}$ bin. To show the fluorescent intensity of Jub or Ecad at vertical or horizontal edges, for each embryo 715 cytoplasmic fluorescence is subtracted from the average fluorescent intensity of edges in the vertical $\left(75-90^{\circ}\right)$ and the horizontal $\left(0-15^{\circ}\right)$ bins.

Analysis of TCJs: Fluorescence within cell vertices (v) was measured using the ellipse tool in Fiji for 30-45 vertices per embryo (e). For enrichment at tricellular junctions, mean intensity of vertices was divided by the mean intensity of bicellular cell edges determined via trajectories drawn in SIESTA. For correlation analysis, ROIs of individual bicellular junctions were subjected to coloc2 in Fiji.

Quantification of Gaps: In Fiji, on an image representing a field of view of $\left(2136.4 \mu^{2}\right)$, the 725 brush tool was used to mask each gap, and then the wand tool was used to measure area of gap and fit ellipse.

Colocalization of signal: To determine the degree of colocalization of Cno versus $\alpha$-Cat construct signal, ROIs of junctions (j) were compared for a number of embryos (e) using the coloc2 plugin on ImageJ. 


\section{Statistics}

735 Prism v9 (GraphPad) was used for statistical analysis and plots, except for the balloon plot in Fig. 4 which was made using Microsoft Excel. In violin plots, bold lines indicate the median, thinner lines represent interquartile ranges. For bar graphs, height of bar indicates mean, and error bars indicate standard deviation. Significance was calculated on measurements from each cell (c), junction (j) or vertex (v) for a number of embryos (e). In general, one-way ANOVA was used to

740 determine significance between the levels of fluorescence in rescue conditions, and Two-way ANOVA was performed on measurements of planar polarity. In experiments with only two conditions, the Mann-Whitney unpaired two-tailed t-test was used. To compare terminal cuticle phenotypes, Chi-square analysis was performed on pairs of conditions. For more details per experiment please refer to Supplemental Table 1. 


\section{Acknowledgements}

We thanks Ritu Sarpal and Limin Wang for providing the data shown in Fig. S1. We thank Mark Peifer for discussion of unpublished data and comments on the manuscript. We thank Nick Brown,

750 Thomas Lecuit, Mark Peifer, the Bloomington Drosophila Stock Center, the Drosophila RNAi Screening Center at Harvard Medical School, and the Developmental Studies Hybridoma Bank for reagents. We like to thank the Imaging Facility of the Department of Cell and Systems Biology, University of Toronto, for support. This work was funded by a project grant from the Canadian Institutes for Health Research (to U.T.). U.T. is a Canada Research Chair for Epithelial Polarity 755 and Development.

\section{Author contributions}

The project was conceived and experiments were designed by L.S. and U.T. L.S. carried out experiments and data analysis. U.T. provided supervision and raised funds. The paper was written 760 by L.S. and U.T.

\section{Competing interest}

The authors declare no competing interests.

\section{Data and material availability}

All data are available in the manuscript or supplementary materials.

\section{Supplementary materials}

Supplemental Figures S1 - S5 


\section{References:}

775

780

785

Abreu-Blanco, M.T., J.M. Verboon, and S.M. Parkhurst. 2014. Coordination of Rho family GTPase activities to orchestrate cytoskeleton responses during cell wound repair. Curr. Biol : CB. 24:144. doi: 10.1016/j.cub.2013.11.048.

Alatortsev, V.E., I.A. Kramerova, M.V. Frolov, S.A. Lavrov, and E.D. Westphal. 1997. Vinculin gene is non-essential in Drosophila melanogaster. FEBS Lett. 413:197-201.

Alégot, H., C. Markosian, C. Rauskolb, J. Yang, E. Kirichenko, Y. Wang, and K.D. Irvine. 2019. Recruitment of Jub by $\alpha$-catenin promotes Yki activity and Drosophila wing growth. $J$. Cell Sci. 132. doi: 10.1242/jcs.222018.

An, Y., G. Xue, Y. Shaobo, D. Mingxi, X. Zhou, W. Yu, T. Ishibashi, L. Zhang, and Y. Yan. 2017. Apical constriction is driven by a pulsatile apical myosin network in delaminating Drosophila neuroblasts. Development. 144:2153-2164.

Angulo-Urarte, A., T. van der Wal, and S. Huveneers. 2020. Cell-cell junctions as sensors and transducers of mechanical forces. Biochimica Et Biophysica Acta (BBA)Biomembranes.:183316.

Barry, A.K., H. Tabdili, I. Muhamed, J. Wu, N. Shashikanth, G.A. Gomez, C.J. Gottardi, J. De Rooij, N. Wang, and D.E. Leckband. 2014. $\alpha$-catenin cytomechanics-role in cadherindependent adhesion and mechanotransduction. J.Cell.Sci. 127:1779-1791.

Barstead, R.J., and R.H. Waterston. 1991. Vinculin is essential for muscle function in the nematode. J.Cell Biol. 114:715-724.

Bertet, C., L. Sulak, and T. Lecuit. 2004. Myosin-dependent junction remodelling controls planar cell intercalation and axis elongation. Nature. 429:667-671.

Blankenship, J.T., S.T. Backovic, J.S. Sanny, O. Weitz, and J.A. Zallen. 2006. Multicellular rosette formation links planar cell polarity to tissue morphogenesis. Dev. Cell. 11:459470.

Boettner, B., P. Harjes, S. Ishimaru, M. Heke, H.Q. Fan, Y. Qin, L. Van Aelst, and U. Gaul. 2003. The AF-6 homolog canoe acts as a Rap1 effector during dorsal closure of the Drosophila embryo. Genetics. 165:159-169.

Bonello, T.T., K.Z. Perez-Vale, K.D. Sumigray, and M. Peifer. 2018. Rap1 acts via multiple mechanisms to position Canoe and adherens junctions and mediate apical-basal polarity establishment. Development. 145.

Borghi, N., M. Sorokina, O.G. Shcherbakova, W.I. Weis, B.L. Pruitt, W.J. Nelson, and A.R. Dunn. 2012. E-cadherin is under constitutive actomyosin-generated tension that is increased at cell-cell contacts upon externally applied stretch. Proc. Natl Acad. Sci. 109:12568-12573.

Brunet, T., A. Bouclet, P. Ahmadi, D. Mitrossilis, B. Driquez, A. Brunet, L. Henry, F. Serman, G. Béalle, and C. Ménager. 2013. Evolutionary conservation of early mesoderm specification by mechanotransduction in Bilateria. Nat. Commun. 4:1-15. 
Buckley, C.D., J. Tan, K.L. Anderson, D. Hanein, N. Volkmann, W.I. Weis, W.J. Nelson, and A.R. Dunn. 2014. The minimal cadherin-catenin complex binds to actin filaments under force. Science. 346.

815 Bush, M., B.M. Alhanshali, S. Qian, C.B. Stanley, W.T. Heller, T. Matsui, T.M. Weiss, I.D. Nicholl, T. Walz, and D.J. Callaway. 2019. An ensemble of flexible conformations underlies mechanotransduction by the cadherin-catenin adhesion complex. Proc. Natl Acad. Sci. 116:21545-21555.

Cavey, M., M. Rauzi, P. Lenne, and T. Lecuit. 2008. A two-tiered mechanism for stabilization and immobilization of E-cadherin. Nature. 453:751-758.

Charras, G., and A.S. Yap. 2018. Tensile forces and mechanotransduction at cell-cell junctions. Curr. Biol. 28:R445-R457.

Choi, H., S. Pokutta, G.W. Cadwell, A.A. Bobkov, L.A. Bankston, R.C. Liddington, and W.I. Weis. 2012. $\alpha$ E-catenin is an autoinhibited molecule that coactivates vinculin. Proc. Natl. Acad. Sci. 109:8576-8581.

Choi, W., K. Jung, K.S. Nelson, M.A. Bhat, G.J. Beitel, M. Peifer, and A.S. Fanning. 2011. The single Drosophila ZO-1 protein Polychaetoid regulates embryonic morphogenesis in coordination with Canoe/afadin and Enabled. Mol.Biol.Cell. 22:2010-2030.

Clarke, D.N., C.J. Lowe, and W. James Nelson. 2019. The cadherin-catenin complex is necessary for cell adhesion and embryogenesis in Nematostella vectensis. Dev. Biol. 447:170-181. doi: 10.1016/j.ydbio.2019.01.007.

Clarke, D.N., and A.C. Martin. 2021. Actin-based force generation and cell adhesion in tissue morphogenesis. Curr. Biol. 31:R667-R680.

Costa, M., W. Raich, C. Agbunag, B. Leung, J. Hardin, and J.R. Priess. 1998. A Putative Catenin-Cadherin System Mediates Morphogenesis of the Caenorhabditis elegans Embryo. J Cell Biol. 141:297-308.

Cox, R.T., C. Kirkpatrick, and M. Peifer. 1996. Armadillo is required for adherens junction assembly, cell polarity, and morphogenesis during Drosophila embryogenesis. J.Cell Biol. 134:133-148.

Desai, R., R. Sarpal, N. Ishiyama, M. Pellikka, M. Ikura, and U. Tepass. 2013. Monomeric $\alpha-$ catenin links cadherin to the actin cytoskeleton. Nat. Cell Biol. 15:261-273. doi: $10.1038 / \mathrm{ncb} 2685$.

Eritano, A.S., C.L. Bromley, A.B. Albero, L. Schütz, F. Wen, M. Takeda, T. Fukaya, M.M. Sami, T. Shibata, S. Lemke, and Y. Wang. 2020. Tissue-Scale Mechanical Coupling Reduces Morphogenetic Noise to Ensure Precision during Epithelial Folding. Dev. Cell. 53:212-228.e12. doi: 10.1016/j.devcel.2020.02.012.

Escobar, D.J., R. Desai, N. Ishiyama, S.S. Folmsbee, M.N. Novak, A.S. Flozak, R.L. Daugherty, R. Mo, D. Nanavati, R. Sarpal, D. Leckband, M. Ikura, U. Tepass, and C.J. Gottardi. 2015. $\alpha$-Catenin phosphorylation promotes intercellular adhesion through a dual-kinase mechanism. J. Cell Sci. 128:1150-1165. doi: 10.1242/jcs.163824.

Fernandez-Gonzalez, R., S. de Matos Simoes, J. Röper, S. Eaton, and J.A. Zallen. 2009. Myosin II dynamics are regulated by tension in intercalating cells. Dev. Cell. 17:736-743. 
Fernandez-Gonzalez, R., and J.A. Zallen. 2011. Oscillatory behaviors and hierarchical assembly of contractile structures in intercalating cells. Phys. Biol. 8:045005.

855 Finer, J.T., R.M. Simmons, and J.A. Spudich. 1994. Single myosin molecule mechanics: piconewton forces and nanometre steps. Nature. 368:113-119.

Foe, V.E. 1989. Mitotic domains reveal early commitment of cells in Drosophila embryos. Development. 107:1-22.

Häcker, U., and N. Perrimon. 1998. DRhoGEF2 encodes a member of the Dbl family of oncogenes and controls cell shape changes during gastrulation in Drosophila. Genes Dev. $12: 274-284$.

Han, M.K.L., E. Hoijman, E. Nöel, L. Garric, J. Bakkers, and J.d. Rooij. 2016. $\alpha$ E-catenindependent mechanotransduction is essential for proper convergent extension in zebrafish. Biol. Open. 5:1461. doi: 10.1242/bio.021378.

865 Han, M.K.L., Van Der Krogt, Gerard NM, and J. De Rooij. 2017. Zygotic vinculin is not essential for embryonic development in zebrafish. PloS One. 12:e0182278.

Harris, T.J., and U. Tepass. 2010. Adherens junctions: from molecules to morphogenesis. Nat. Rev. Mol. Cell Biol. 11:502-514.

Huang, J., W. Zhou, W. Dong, A.M. Watson, and Y. Hong. 2009. Directed, efficient, and versatile modifications of the Drosophila genome by genomic engineering. Proc. Natl. Acad. Sci. 106:8284-8289.

Huveneers, S., J. Oldenburg, E. Spanjaard, G. van der Krogt, I. Grigoriev, A. Akhmanova, H. Rehmann, and J. de Rooij. 2012. Vinculin associates with endothelial VE-cadherin junctions to control force-dependent remodeling. J.Cell Biol. 196:641-652.

875 Ikeda, W., H. Nakanishi, J. Miyoshi, K. Mandai, H. Ishizaki, M. Tanaka, A. Togawa, K. Takahashi, H. Nishioka, and H. Yoshida. 1999. Afadina key molecule essential for structural organization of cell-cell junctions of polarized epithelia during embryogenesis. J.Cell Biol. 146:1117-1132.

Ishiyama, N., and M. Ikura. 2012. The three-dimensional structure of the cadherin-catenin complex. In Adherens Junctions: From Molecular Mechanisms to Tissue Development and Disease. Springer. 39-62.

Ishiyama, N., R. Sarpal, M.N. Wood, S.K. Barrick, T. Nishikawa, H. Hayashi, A.B. Kobb, A.S. Flozak, A. Yemelyanov, and R. Fernandez-Gonzalez. 2018. Force-dependent allostery of the $\alpha$-catenin actin-binding domain controls adherens junction dynamics and functions. Nat. Commun. 9:1-17.

Ishiyama, N., N. Tanaka, K. Abe, Y.J. Yang, Y.M. Abbas, M. Umitsu, B. Nagar, S.A. Bueler, J.L. Rubinstein, and M. Takeichi. 2013. An autoinhibited structure of $\alpha$-catenin and its implications for vinculin recruitment to adherens junctions. J.Biol.Chem. 288:1591315925.

890 Jurado, J., J. de Navascués, and N. Gorfinkiel. 2016. $\alpha$-Catenin stabilises Cadherin-Catenin complexes and modulates actomyosin dynamics to allow pulsatile apical contraction. $J$. Cell Sci. 129:4496-4508. doi: 10.1242/jcs. 193268. 
Jürgens, G., E. Wieschaus, C. Nüsslein-Volhard, and H. Kluding. 1984. Mutations affecting the pattern of the larval cuticle inDrosophila melanogaster. Roux's Arch. Dev. Biol. 193:283295.

Kale, G.R., X. Yang, J. Philippe, M. Mani, P. Lenne, and T. Lecuit. 2018. Distinct contributions of tensile and shear stress on E-cadherin levels during morphogenesis. Nat. Commun. 9:1-16.

Kim, T., S. Zheng, J. Sun, I. Muhamed, J. Wu, L. Lei, X. Kong, D.E. Leckband, and Y. Wang. 2015. Dynamic visualization of $\alpha$-catenin reveals rapid, reversible conformation switching between tension states. Curr. Biol. 25:218-224.

Klapholz, B., S.L. Herbert, J. Wellmann, R. Johnson, M. Parsons, and N.H. Brown. 2015. Alternative mechanisms for talin to mediate integrin function. Curr. Biol. 25:847-857.

Kofron, M., A. Spagnuolo, M. Klymkowsky, C. Wylie, and J. Heasman. 1997. The roles of maternal alpha-catenin and plakoglobin in the early Xenopus embryo. Development. 124:1553.

Ladoux, B., W.J. Nelson, J. Yan, and R.M. Mege. 2015. The mechanotransduction machinery at work at adherens junctions. Integr. Biol. 7:1109-1119.

Le Duc, Q., Q. Shi, I. Blonk, A. Sonnenberg, N. Wang, D. Leckband, and J. De Rooij. 2010. Vinculin potentiates E-cadherin mechanosensing and is recruited to actin-anchored sites
within adherens junctions in a myosin II-dependent manner. J.Cell Biol. 189:1107-1115.

Leckband, D.E., and J. De Rooij. 2014. Cadherin adhesion and mechanotransduction. Annu.Rev.Cell Dev.Biol. 30:291-315.

Lecuit, T., and A.S. Yap. 2015. E-cadherin junctions as active mechanical integrators in tissue dynamics. Nat.Cell Biol. 17:533-539.

Levayer, R., and T. Lecuit. 2013a. Oscillation and polarity of E-cadherin asymmetries control actomyosin flow patterns during morphogenesis. Dev. Cell. 26:162-175.

Levayer, R., and T. Lecuit. 2013b. Oscillation and Polarity of E-Cadherin Asymmetries Control Actomyosin Flow Patterns during Morphogenesis. Dev. Cell. 26:162-175. doi: 10.1016/j.devcel.2013.06.020.

Li, J., J. Newhall, N. Ishiyama, C. Gottardi, M. Ikura, D.E. Leckband, and E. Tajkhorshid. 2015. Structural determinants of the mechanical stability of $\alpha$-catenin. J.Biol.Chem. 290:1889018903.

Lin, C., J. Xu, W. Yang, C. Wang, Y. Li, L. Cheng, L. Zhang, and J. Hsu. 2017. Smurf Downregulates Echinoid in the Amnioserosa To Regulate Drosophila Dorsal Closure. Genetics. 206:985-992. doi: 10.1534/genetics.116.196527.

Lowe, N., J.S. Rees, J. Roote, E. Ryder, I.M. Armean, G. Johnson, E. Drummond, H. Spriggs, J. Drummond, and J.P. Magbanua. 2014. Analysis of the expression patterns, subcellular localisations and interaction partners of Drosophila proteins using a pigP protein trap library. Development. 141:3994-4005.

Lye, C.M., H.W. Naylor, and B. Sanson. 2014. Subcellular localisations of the CPTI collection of YFP-tagged proteins in Drosophila embryos. Development. 141:4006-4017. 
Maartens, A.P., J. Wellmann, E. Wictome, B. Klapholz, H. Green, and N.H. Brown. 2016. Drosophila vinculin is more harmful when hyperactive than absent, and can circumvent integrin to form adhesion complexes. J.Cell.Sci. 129:4354-4365.

Maître, J., and C. Heisenberg. 2013. Three functions of cadherins in cell adhesion. Curr. Biol. 23:R626-R633.

Maki, K., S. Han, Y. Hirano, S. Yonemura, T. Hakoshima, and T. Adachi. 2016. Mechanoadaptive sensory mechanism of $\alpha$-catenin under tension. Sci. Rep. 6:24878. doi: $10.1038 /$ srep24878.

Manning, L.A., K.Z. Perez-Vale, K.N. Schaefer, M.T. Sewell, and M. Peifer. 2019. The Drosophila Afadin and ZO-1 homologues Canoe and Polychaetoid act in parallel to maintain epithelial integrity when challenged by adherens junction remodeling. Mol.Biol.Cell. 30:1938-1960.

Marie, H., S.J. Pratt, M. Betson, H. Epple, J.T. Kittler, L. Meek, S.J. Moss, S. Troyanovsky, D. Attwell, and G.D. Longmore. 2003. The LIM protein Ajuba is recruited to cadherindependent cell junctions through an association with $\alpha$-catenin. J.Biol.Chem. 278:12201228.

Martin, A.C. 2020. The physical mechanisms of Drosophila gastrulation: mesoderm and endoderm invagination. Genetics. 214:543-560.

Martin, A.C., M. Gelbart, R. Fernandez-Gonzalez, M. Kaschube, and E.F. Wieschaus. 2010. Integration of contractile forces during tissue invagination. J Cell Biol. 188:735-749. doi: 10.1083/jcb.200910099.

Martino, F., A.R. Perestrelo, V. Vinarský, S. Pagliari, and G. Forte. 2018. Cellular mechanotransduction: from tension to function. Fron. Physiol. 9:824.

Matsuzawa, K., T. Himoto, Y. Mochizuki, and J. Ikenouchi. 2018. $\alpha$-Catenin controls the anisotropy of force distribution at Cell-Cell junctions during collective cell migration. Cell Rep. 23:3447-3456.

Mège, R.M., and N. Ishiyama. 2017. Integration of cadherin adhesion and cytoskeleton at adherens junctions. Cold Spring Harb. Perspect. Biol. 9:a028738.

Miller, K.G., C.M. Field, and B.M. Alberts. 1989. Actin-binding proteins from Drosophila embryos: a complex network of interacting proteins detected by F-actin affinity chromatography. J.Cell Biol. 109:2963-2975.

Munjal, A., J. Philippe, E. Munro, and T. Lecuit. 2015. A self-organized biomechanical network drives shape changes during tissue morphogenesis. Nature. 524:351-355.

Nieset, J.E., A.R. Redfield, F. Jin, K.A. Knudsen, K.R. Johnson, and M.J. Wheelock. 1997. Characterization of the interactions of alpha-catenin with alpha-actinin and betacatenin/plakoglobin. J.Cell.Sci. 110:1013-1022.

Paré, A.C., and J.A. Zallen. 2020. Cellular, molecular, and biophysical control of epithelial cell intercalation. Curr. Top. Dev. Biol. 136:167-193. 
Perez-Vale, K., K. Yow, A. Byrnes, T. Finegan, K.C. Slep, and M. Peifer. 2021. Multivalent interactions make adherens junction-cytoskeletal linkage robust during morphogenesis. bioRxiv.

Pinheiro, D., and Y. Bellaïche. 2018. Mechanical force-driven adherens junction remodeling and epithelial dynamics. Dev. Cell. 47:3-19.

Pokutta, S., F. Drees, Y. Takai, W.J. Nelson, and W.I. Weis. 2002. Biochemical and structural definition of the 1-afadin-and actin-binding sites of $\alpha$-catenin. J.Biol.Chem. 277:1886818874.

Pratt, S.J., H. Epple, M. Ward, Y. Feng, V.M. Braga, and G.D. Longmore. 2005. The LIM protein Ajuba influences p130Cas localization and Rac1 activity during cell migration. J.Cell Biol. 168:813-824.

Rangarajan, E.S., and T. Izard. 2012. The cytoskeletal protein $\alpha$-catenin unfurls upon binding to vinculin. J.Biol.Chem. 287:18492-18499.

Rauskolb, C., E. Cervantes, F. Madere, and K.D. Irvine. 2019. Organization and function of tension-dependent complexes at adherens junctions. J.Cell.Sci. 132.

Rauskolb, C., S. Sun, G. Sun, Y. Pan, and K.D. Irvine. 2014. Cytoskeletal tension inhibits Hippo signaling through an Ajuba-Warts complex. Cell. 158:143-156.

Rauzi, M., P. Lenne, and T. Lecuit. 2010. Planar polarized actomyosin contractile flows control epithelial junction remodelling. Nature. 468:1110-1114.

990 Razzell, W., M.E. Bustillo, and J.A. Zallen. 2018. The force-sensitive protein Ajuba regulates cell adhesion during epithelial morphogenesis. J. Cell Biol. 217:3715. doi: 10.1083/jcb.201801171.

Rimm, D.L., E.R. Koslov, P. Kebriaei, C.D. Cianci, and J.S. Morrow. 1995. Alpha 1 (E)-catenin is an actin-binding and-bundling protein mediating the attachment of F-actin to the membrane adhesion complex. Proc. Natl. Acad. Sci. 92:8813-8817.

Rothenberg, K.E., and R. Fernandez-Gonzalez. 2019. Forceful closure: cytoskeletal networks in embryonic wound repair. Mol. Biol. Cell. 30:1353-1358.

Sabino, D., N.H. Brown, and R. Basto. 2011. Drosophila Ajuba is not an Aurora-A activator but is required to maintain Aurora-A at the centrosome. J. Cell. Sci. 124:1156-1166.

1000 Sakakibara, S., K. Mizutani, A. Sugiura, A. Sakane, T. Sasaki, S. Yonemura, and Y. Takai. 2020. Afadin regulates actomyosin organization through $\alpha$ E-catenin at adherens junctions. $J$. Cell Biol. 219.

Sarpal, R., V. Yan, L. Kazakova, L. Sheppard, J.C. Yu, R. Fernandez-Gonzalez, and U. Tepass. $2019 \mathrm{~b}$. Role of $\alpha$-Catenin and its mechanosensing properties in regulating Hippo/YAPdependent tissue growth. PLOS Genet. 15:e1008454. doi: 10.1371/journal.pgen.1008454.

Sarpal, M. Pellikka, R.R. Patel, F.Y.W. Hui, D. Godt, and U. Tepass. 2012. Mutational analysis supports a core role for Drosophila -Catenin in adherens junction function. J. Cell Sci. 125:233-245. doi: 10.1242/jcs.096644. 
Sawyer, J.K., N.J. Harris, K.C. Slep, U. Gaul, and M. Peifer. 2009. The Drosophila afadin homologue Canoe regulates linkage of the actin cytoskeleton to adherens junctions during apical constriction. J. Cell Biol. 186:57-73. doi: 10.1083/jcb.200904001.

Sawyer, J.K., W. Choi, K. Jung, L. He, N.J. Harris, and M. Peifer. 2011. A contractile actomyosin network linked to adherens junctions by Canoe/afadin helps drive convergent extension. Mol. Biol. Cell. 22:2491-2508.

1015 Schepis, A., D. Sepich, and W.J. Nelson. 2012. $\alpha$ E-catenin regulates cell-cell adhesion and membrane blebbing during zebrafish epiboly. Development. 139:537. doi: 10.1242/dev.073932.

Schindelin, J., I. Arganda-Carreras, E. Frise, V. Kaynig, M. Longair, T. Pietzsch, S. Preibisch, C. Rueden, S. Saalfeld, and B. Schmid. 2012. Fiji: an open-source platform for biologicalimage analysis. Nat. Methods. 9:676-682.

Seddiki, R., Narayana, Gautham Hari Narayana Sankara, P. Strale, H.E. Balcioglu, G. Peyret, M. Yao, A.P. Le, C. Teck Lim, J. Yan, and B. Ladoux. 2018. Force-dependent binding of vinculin to $\alpha$-catenin regulates cell-cell contact stability and collective cell behavior. Mol. Biol. Cell. 29:380-388.

1025 Simões, S., Y. Oh, M.F. Wang, R. Fernandez-Gonzalez, and U. Tepass. 2017. Myosin II promotes the anisotropic loss of the apical domain during Drosophila neuroblast ingression. J. Cell Biol. 216:1387-1404.

Stephenson, R.E., T. Higashi, I.S. Erofeev, T.R. Arnold, M. Leda, A.B. Goryachev, and A.L. Miller. 2019. Rho flares repair local tight junction leaks. Dev. Cell. 48:445-459. e5.

1030 Takahashi, K., T. Matsuo, T. Katsube, R. Ueda, and D. Yamamoto. 1998. Direct binding between two PDZ domain proteins Canoe and ZO-1 and their roles in regulation of the jun N-terminal kinase pathway in Drosophila morphogenesis. Mech.Dev. 78:97-111.

Takeichi, M. 2018. Historical review of the discovery of cadherin, in memory of Tokindo Okada. Dev.Growth Differ. 60:3-13.

1035 Takeichi, M. 2014. Dynamic contacts: rearranging adherens junctions to drive epithelial remodelling. Nat. Rev. Mol. Cell Biol. 15:397-410.

Tepass, U., E. Gruszynski-DeFeo, T.A. Haag, L. Omatyar, T. Török, and V. Hartenstein. 1996. shotgun encodes Drosophila E-cadherin and is preferentially required during cell rearrangement in the neurectoderm and other morphogenetically active epithelia. Genes

1040 Dev. 10:672-685.

Terekhova, K., S. Pokutta, Y.S. Kee, J. Li, E. Tajkhorshid, G. Fuller, A.R. Dunn, and W.I. Weis. 2019. Binding partner-and force-promoted changes in $\alpha$ E-catenin conformation probed by native cysteine labeling. Sci Rep. 9:1-13.

Tetley, R.J., G.B. Blanchard, A.G. Fletcher, R.J. Adams, and B. Sanson. 2016. Unipolar

1045 distributions of junctional Myosin II identify cell stripe boundaries that drive cell intercalation throughout Drosophila axis extension. Elife. 5:e12094.

Thomas, W.A., C. Boscher, Y. Chu, D. Cuvelier, C. Martinez-Rico, R. Seddiki, J. Heysch, B. Ladoux, J.P. Thiery, and R. Mege. 2013. $\alpha$-Catenin and vinculin cooperate to promote high E-cadherin-based adhesion strength. J.Biol.Chem. 288:4957-4969. 
1050 Torres, M., A. Stoykova, O. Huber, K. Chowdhury, P. Bonaldo, A. Mansouri, S. Butz, R. Kemler, and P. Gruss. 1997. An $\alpha$-E-catenin gene trap mutation defines its function in preimplantation development. Proc. Natl. Acad. Sci. 94:901-906.

Twiss, F., Q. le Duc, S. van der Horst, H. Tabdili, G. Van Der Krogt, N. Wang, H. Rehmann, S. Huveneers, D.E. Leckband, and J. de Rooij. 2012. Vinculin-dependent Cadherin mechanosensing regulates efficient epithelial barrier formation. Biol. Open. 1:1128-1140. doi: 10.1242/bio.20122428.

Vanderleest, T.E., C.M. Smits, Y. Xie, C.E. Jewett, J.T. Blankenship, and D. Loerke. 2018. Vertex sliding drives intercalation by radial coupling of adhesion and actomyosin networks during Drosophila germband extension. Elife. 7:e34586.

1060 Wang, Y., Z. Khan, and E.F. Wieschaus. 2013. Distinct Rap1 activity states control the extent of epithelial invagination via $\alpha$-catenin. Dev. Cell. 25:299-309.

Wei, S., L.M. Escudero, F. Yu, L. Chang, L. Chen, Y. Ho, C. Lin, C. Chou, W. Chia, and J. Modolell. 2005. Echinoid is a component of adherens junctions that cooperates with DECadherin to mediate cell adhesion. Dev. Cell. 8:493-504.

1065 Witzel, H.R., B. Jungblut, C.P. Choe, J.G. Crump, T. Braun, and G. Dobreva. 2012. The LIM protein Ajuba restricts the second heart field progenitor pool by regulating Is11 activity. Dev. Cell. 23:58-70.

$\mathrm{Xu}, \mathrm{W} ., \mathrm{H}$. Baribault, and E.D. Adamson. 1998. Vinculin knockout results in heart and brain defects during embryonic development. Development. 125:327-337.

1070 Xu, X., S. Pokutta, M. Torres, M.F. Swift, D. Hanein, N. Volkmann, and W.I. Weis. 2020. Structural basis of $\alpha$ E-catenin-F-actin catch bond behavior. Elife. 9:e60878.

Yamada, S., S. Pokutta, F. Drees, W.I. Weis, and W.J. Nelson. 2005. Deconstructing the cadherin-catenin-actin complex. Cell. 123:889-901.

Yao, M., W. Qiu, R. Liu, A.K. Efremov, P. Cong, R. Seddiki, M. Payre, C.T. Lim, B. Ladoux, and R. Mege. 2014. Force-dependent conformational switch of $\alpha$-catenin controls vinculin binding. Nat. Commun. 5:1-12.

Yonemura, S., Y. Wada, T. Watanabe, A. Nagafuchi, and M. Shibata. 2010. $\alpha$-Catenin as a tension transducer that induces adherens junction development. Nat.Cell Biol. 12:533542.

1080 Yu, H.H., and J.A. Zallen. 2020. Abl and Canoe/Afadin mediate mechanotransduction at tricellular junctions. Science. 370.

Zallen, J.A., and J.T. Blankenship. 2008. Multicellular dynamics during epithelial elongation. Semin. Cell Dev. Biol. 19:263-270.

Zallen, J.A., and E. Wieschaus. 2004. Patterned gene expression directs bipolar planar polarity in 1085 Drosophila. Dev. Cell. 6:343-355.

Zhadanov, A.B., D.W. Provance Jr, C.A. Speer, J.D. Coffin, D. Goss, J.A. Blixt, C.M. Reichert, and J.A. Mercer. 1999. Absence of the tight junctional protein AF-6 disrupts epithelial cell-cell junctions and cell polarity during mouse development. Curr. Biol. 9:880-S2. 
bioRxiv preprint doi: https://doi org/101101/2021.09.08.459511. this version posted September 9, 2021. The copyright holder for this preprint

(which was not certified by peer review) is the author/funder, who has granted bioRxiv a license to display the preprint in perpetuity. It is made available under aCC-BY-NC-ND 4.0 International license.

Zirin, J., Y. Hu, L. Liu, D. Yang-Zhou, R. Colbeth, D. Yan, B. Ewen-Campen, R. Tao, E. Vogt, and S. VanNest. 2020. Large-scale transgenic Drosophila resource collections for lossand gain-of-function studies. Genetics. 214:755-767. 


\section{Figure Legends}

Figure 1. The $\alpha$-Cat $M$ region is required for epithelial integrity during embryogenesis.

(A,B) Schematic of $\alpha$-Cat under no/low and high tension. See text for further discussion.

(C) Quantification of cuticle defects of embryos expressing $\alpha$-CatR or $\alpha$-Cat deletion constructs

1100 in an $\alpha$-Cat-RNAi background.

(D) Representative cuticle images of embryos of the indicated genotypes. False color shading of cuticle in blue and denticle belts in yellow. Pink arrowheads point to abdominal denticle belts that were used for quantification in (C). Scale bar, $100 \mu \mathrm{m}$.

1105 Figure 2. The $\alpha$-Cat $M$ region is essential for adhesion during mesoderm invagination and germband extension.

(A) Quantification of mesoderm invagination defects in embryos of indicated genotype. Color coded example images are given at right. Orange lines indicate edges of mesoderm. Red - open ventral furrow; Yellow - partially fused ventral furrow; Indigo - $>90 \%$ midline fusion. Scale bar;

$1110100 \mu \mathrm{m}$.

(B) Quantification of defects in the lateral ectoderm during germband extension in embryos of indicated genotype. Classification used: Indigo - wild type, very few gaps seen; Cyan - small gaps; Yellow - large gaps or tears; Red - few if any identifiable AJs. Pink areas represent regions of gaps where apical junctions have lost contact. Scale bar, $10 \mu \mathrm{m}$.

1115 (C) Area of gaps in $\alpha$-Cat-RNAi $\alpha$-CatX embryos.

(D) Plot showing the angle of the major axis of ellipses fitted to each gap in $\alpha$-Cat-RNAi $\alpha$-CatX embryos. $\mathrm{N}=$ number of embryos(e)

(E) Extension of the germband observed over 40 minutes in $\alpha$-Cat-RNAi $\alpha$-CatX embryos. For

(C) and (E) significance given by ordinary one-way ANOVA, $(* * * *=\mathrm{P}<0.0001$, $* * *=\mathrm{P}$ 
1125 Figure 3. Myosin distribution and Rho1 activity in embryos expressing $\alpha$-Cat M region deletions.

(A) Representative images of ectoderm during germband extension of $\alpha$-Cat-RNAi $\alpha$-CatX embryos. Myosin cables are highlighted with orange lines in the right duplicate images.

(B) Series of stills from a live $\alpha$-Cat-RNAi $\alpha$-Cat- $\triangle M 23$ embryo showing gaps forming at

1130 vertical edges (arrowheads) with accumulations of myosin at gap perimeters.

(C) Stills from live $\alpha$-Cat-RNAi $\alpha$-CatX embryos expressing the Rho1 activity probe, AniRBD::GFP. Note accumulation of Ani-RBD::GFP in gap areas (arrowheads).

(D) Series of stills from a live $\alpha$-Cat-RNAi $\alpha$-Cat- $\triangle M 23$ embryo showing enrichment of AniRBD::GFP at a forming gap between cells (arrowheads). Cell contacts first split and

1135 subsequently Ani-RBD signal increases.

(E) Quantification of myosin $\alpha$-Cat-RNAi $\alpha$-CatX embryos.

(F) Quantification of Ani-RBD::GFP signal at cortices of cells which are not in contact with a gap. Average FI of Ani-RBD::GFP signal at the perimeter of cells is compared to the perimeter of gaps. For (E) and (F) significance is calculated by ordinary one-way ANOVA (**** $=\mathrm{P}$

$1140<0.0001, * * *=\mathrm{P}<0.0002, * *=\mathrm{P}<0.0021) . \mathrm{N}=$ number of cells (c), and embryos (e).. Scale bar in (A-D), $10 \mu \mathrm{m}$.

\section{Figure 4. The $M$ region regulates the levels of $\mathrm{AJ}$ components.}

(A) Stills of live $\alpha$-Cat-RNAi $\alpha$-CatX embryos at stage 8 expressing Ecad::GFP and

1145 Vinc::mCherry.

(B) Stills of live $\alpha$-Cat-RNAi $\alpha$-CatX embryos at stage 8 expressing Jub::GFP. Scale bar for (A) and $(\mathrm{B}), 10 \mu \mathrm{m}$.

(C) Fluorescent intensities (FI) of junctional signal of Vinc::mCherry, Ecad::GFP, and Jub::GFP in $\alpha$-Cat-RNAi $\alpha$-CatX embryos at stage $8 . \mathrm{N}=$ number of cells (c), and embryos (e). The ratio of

1150 cortical Vinc to Ecad per cell is given as Vinc/Ecad. Significance calculated by ordinary oneway ANOVA $(* * * *=\mathrm{P}<0.0001, *=\mathrm{P}<0.0332)$.

(D) Balloon plot summarizing fold changes of junctional fluorescent signals.

(E) Schematic illustration of the functional relationship between M1, M2, and M3 in respect to the recruitment of Vinc to the AJ. 


\section{Figure 5. M region supports mechanosensitive recruitment of Jub to AJs}

(A) Stills from live $\alpha$-Cat-RNAi $\alpha$-CatX embryos expressing Jub::GFP. Scale bar, $10 \mu \mathrm{m}$.

(B,C) The average fluorescent intensity (FI) of Jub (with background cytoplasmic signal subtracted) for $\alpha$-Cat-RNAi $\alpha$-CatX embryos at stage 8 is plotted for (B) horizontal edges $\left(0-15^{\circ}\right)$

1160 and (C) vertical edges $\left(75-90^{\circ}, 0=\right.$ anterior-posterior axis). $\mathrm{N}=$ number of embryos (e).

(D) Quantification of planar polarity of Jub is plotted per embryo as average FI of vertical edges divided by that of horizontal edges. The ratio in (D) is derived from the data in (B) and (C). For (B) and (C), significance is calculated by ordinary one-way ANOVA, and for (D) by two-way ANOVA $(* * * *=\mathrm{P}<0.0001, * * *=\mathrm{P}<0.0002, * *=\mathrm{P}<0.0021, *=\mathrm{P}<0.0332)$. See Fig S3 for more

1165 detailed breakdown of enrichment of fluorescent intensity by angles of edges.

(E) Analysis of cuticle defects of $\alpha$-Cat-RNAi $\alpha$-CatX embryos and either GFP-RNAi as a control or Jub-RNAi. Chi-square test $(* * * *=\mathrm{P}<0.0001)$.

\section{Figure 6. The M1-Vinc interaction supports Ecad stability and reinforces adhesion.}

1170 (A) Ecad planar polarity (enrichment at vertical edges) is shown in $\alpha$-Cat-RNAi $\alpha$-CatX embryos at stage 8 expressing either GAP43::mCherry or Vinc::mCherry.

(B) An extra copy of Vinc (Vinc::mCherry) improves adhesion in the lateral ectoderm in $\alpha$-CatRNAi $\alpha$-Cat- $\triangle M 23$ embryos at stage 8.

(C, D) Comparison of control and Vinc-CO embryos expressing Ecad::GFP. Stills from live

1175 embryos at stage 8. Fluorescent intensity (FI) of Ecad and Ecad planar polarity (D).

(E) Denticle belt count of $\alpha$-Cat-RNAi $\alpha$-Cat- $\Delta M 23$ embryos with and without a Vinc maternalzygotic (MZ) null mutant background.

(F) Live imaged stills comparing Jub::GFP signal in wildtype and a Vinc MZ null mutant embryo at stage 8 .

1180 (G) $\alpha$-Cat-RNAi $\alpha$-CatR- $\Delta M 1$ embryos at stage 8 show less uniform junctional distribution of Ecad::GFP compared to $\alpha$-Cat-RNAi $\alpha$-CatR controls as indicated by the increased normalized standard deviation (SD) of Ecad::GFP signal at AJs.

(H) Junctional $\alpha$-Cat::YFP signal (same color map in F), in stage 8 embryos is reduced and less uniformly distributed in the absence of Vinc, as indicated by an increase of the normalized SD of $\alpha$-Cat::YFP signal. 
$\mathrm{N}=$ junctions (j) and embryos (e) in (G) and (I). $\mathrm{N}=$ cells (c) and embryos (e) in (E) and (K).

Significance is calculated by two way ANOVA for (A), Chi-square for (E), and Mann-Whitney two-tailed test for (B), (C), (D), (F), (G), and (H) (**** $=\mathrm{P}<0.0001, * * *=\mathrm{P}<0.0002$, $* *=\mathrm{P}<0.0021, *=\mathrm{P}<0.0332)$.

Figure 7. Mechanosensitive recruitment of Cno to TCJs requires the $\alpha$-Cat $M$ region.

(A) Stills of lateral ectoderm at stage 8 of $\alpha$-Cat-RNAi $\alpha$-CatX live embryos expressing Cno::YFP. Orange arrowheads in close-ups at right point to TJCs.

(B) Cortical levels of Cno of $\alpha$-Cat-RNAi $\alpha$-CatX embryos. $\mathrm{N}=$ number of embryos (e) and cells 1195 (c).

(C) Levels of Cno measured at intact TCJs in $\alpha$-Cat-RNAi $\alpha$-CatX embryos. $\mathrm{N}=$ number of embryos (e) and vertices (v).

(D) Ratio of the mean fluorescence of Cno at TCJs versus bicellular junctions (BCJs) is plotted per embryo. Significance is given by one-way ANOVA $(* * * *=\mathrm{P}<0.0001, * * *=\mathrm{P}<0.0002$, $1200 * *=\mathrm{P}<0.0021, *=\mathrm{P}<0.0332)$.

\section{Figure 8. Cno supports Ecad stability in a parallel pathway to the $\alpha$-Cat $M$ region}

(A) Quantification of cuticle defects from cno-RNAi $\alpha$-Cat-RNAi $\alpha$-CatX and gfp-RNAi $\alpha$-CatRNAi $\alpha$-CatX embryos. Phenotypic categories are color-coded and examples are given in Fig. S5.

1205 (B) Overexpression of Cno improves epithelial integrity in $\alpha$-Cat-RNAi embryos.

(C) Denticle belt count for $\alpha$-Cat-RNAi $\alpha$-CatX embryos overexpressing Cno or GFP-RNAi as control. $g f p-R N A i$ controls same as in Fig. 5E.

(D) Ectoderm at stage 8/9 of $\alpha$-Cat-RNAi $\alpha$-CatX embryos expressing either UAS-cno, cno-RNAi or $U A S-g f p-R N A i$ as control immunostained for Ecad. Ecad levels are enhanced by Cno

1210 overexpression, ameliorating defects found in $\alpha$-Cat- $\Delta \mathrm{M} 23$ embryos, whereas Cno knockdown causes an enhancement of $\mathrm{AJ}$ fragmentation in $\alpha$-CatR- $\Delta \mathrm{M} 1$ embryos compared to control.

(E) Rap1-RNAi enhances defects in $\alpha$-Cat-RNAi $\alpha-C a t R$ and $\alpha$-Cat-RNAi $\alpha-C a t-\Delta M$ embryos. $g F P-R N A i$ controls same as in (C). Chi-square test, $* * * *=\mathrm{P}<0.0001$.

(F) Vinc null mutant enhances defects caused by cno-RNAi. Chi-square test, $* * * *=\mathrm{P}<0.0001$.

1215 (G) $j u b-R N A i$ enhances defects caused by cno-RNAi. Chi-square test, ${ }^{* * *}=\mathrm{P}<0.0002$. 
Figure 9. Model of $\alpha$-Cat $M$ region mechanosensing in the Drosophila ectoderm during germband extension.

(A) Schematic illustrating three distinct conformation of the $\alpha$-Cat M-region found at low,

1220 medium and high tension cell contacts. demonstrating the distinct contributions of M-region subdomains to the recruitment of binding partners. M-region subdomains have an inhibitory effect on each other.

(B) Diagram illustrating the tension landscape in the ectoderm during germband extension, showing low tension contacts (horizontal edges), medium tension contacts (vertical edges) and 1225 high tension contacts (TCJs/vertices).

(C) Schematic illustration of anticipated distribution of the three $\alpha$-Cat conformation highlighted in (A) in wild-type, $\alpha$-Cat-RNAi $\alpha$-CatR- $\triangle M 1$ embryos and $\alpha$-Cat-RNAi $\alpha$-Cat- $\Delta M 2$. See text for further discussion. 


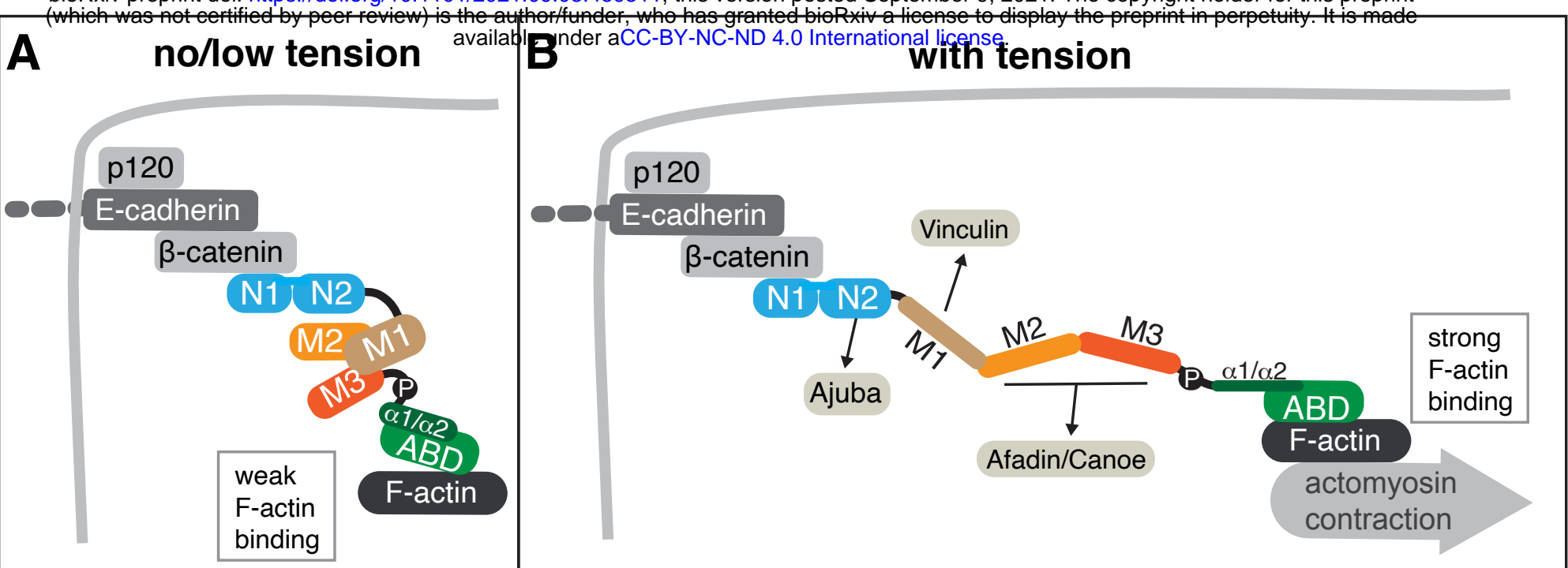

C

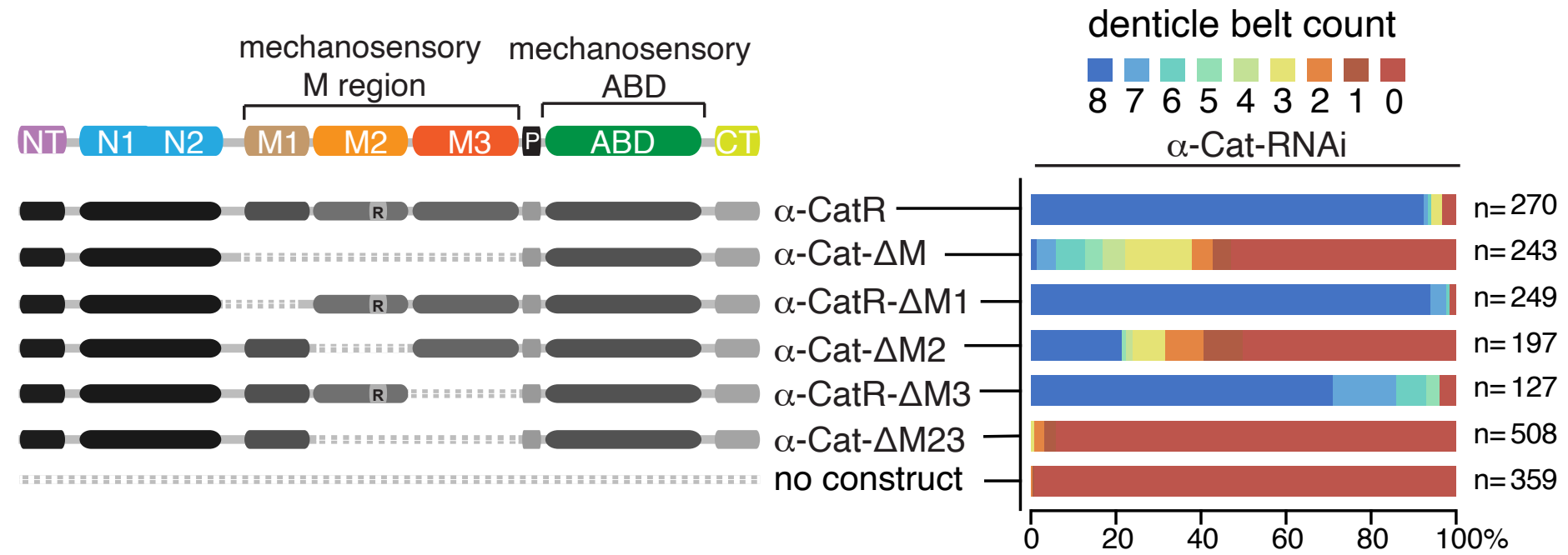

D

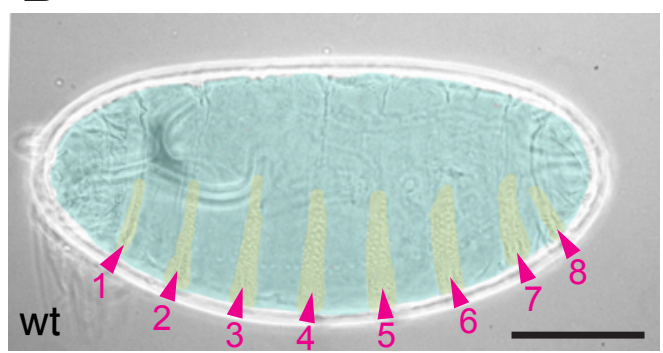

a-Cat-RNAi
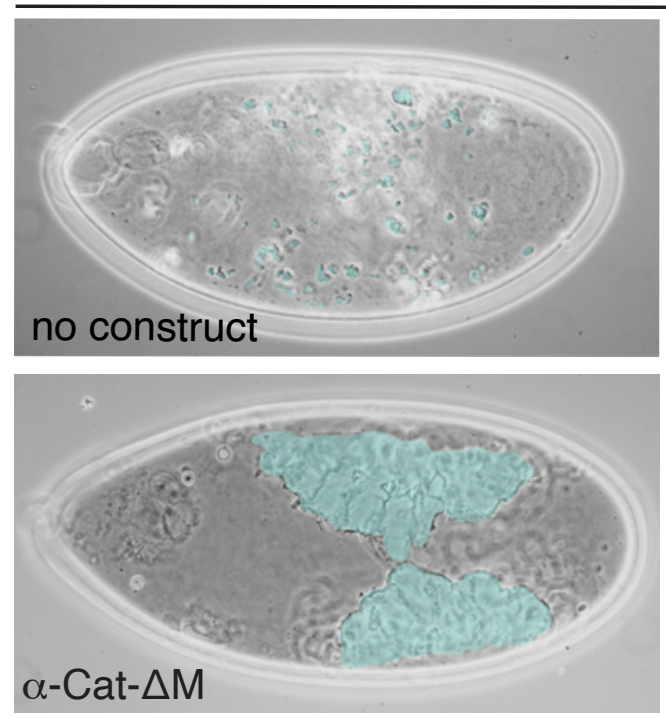

$\alpha$-CatR

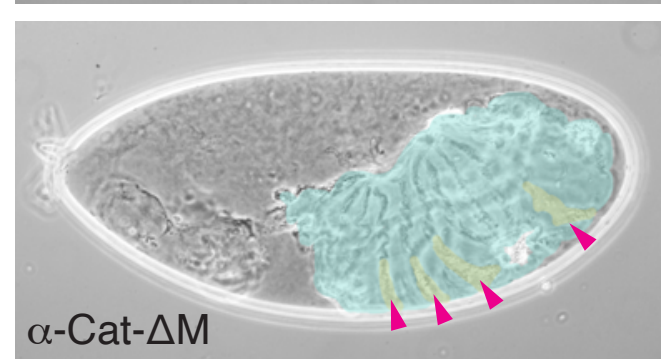




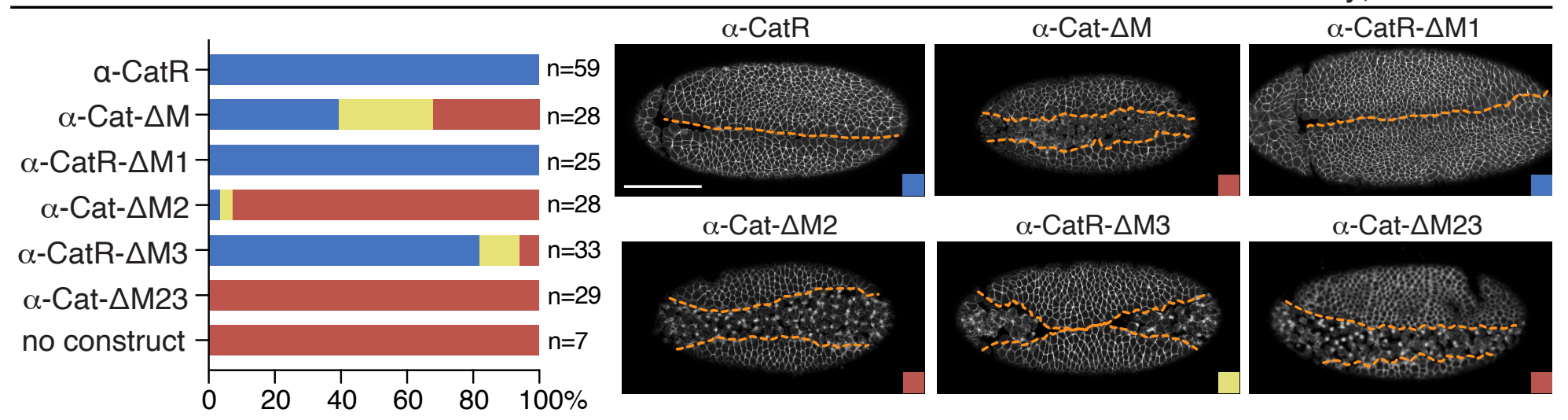

B Ectoderm defects

Ecad::GFP, GAP43::mCherry; $\alpha$-Cat-RNAi
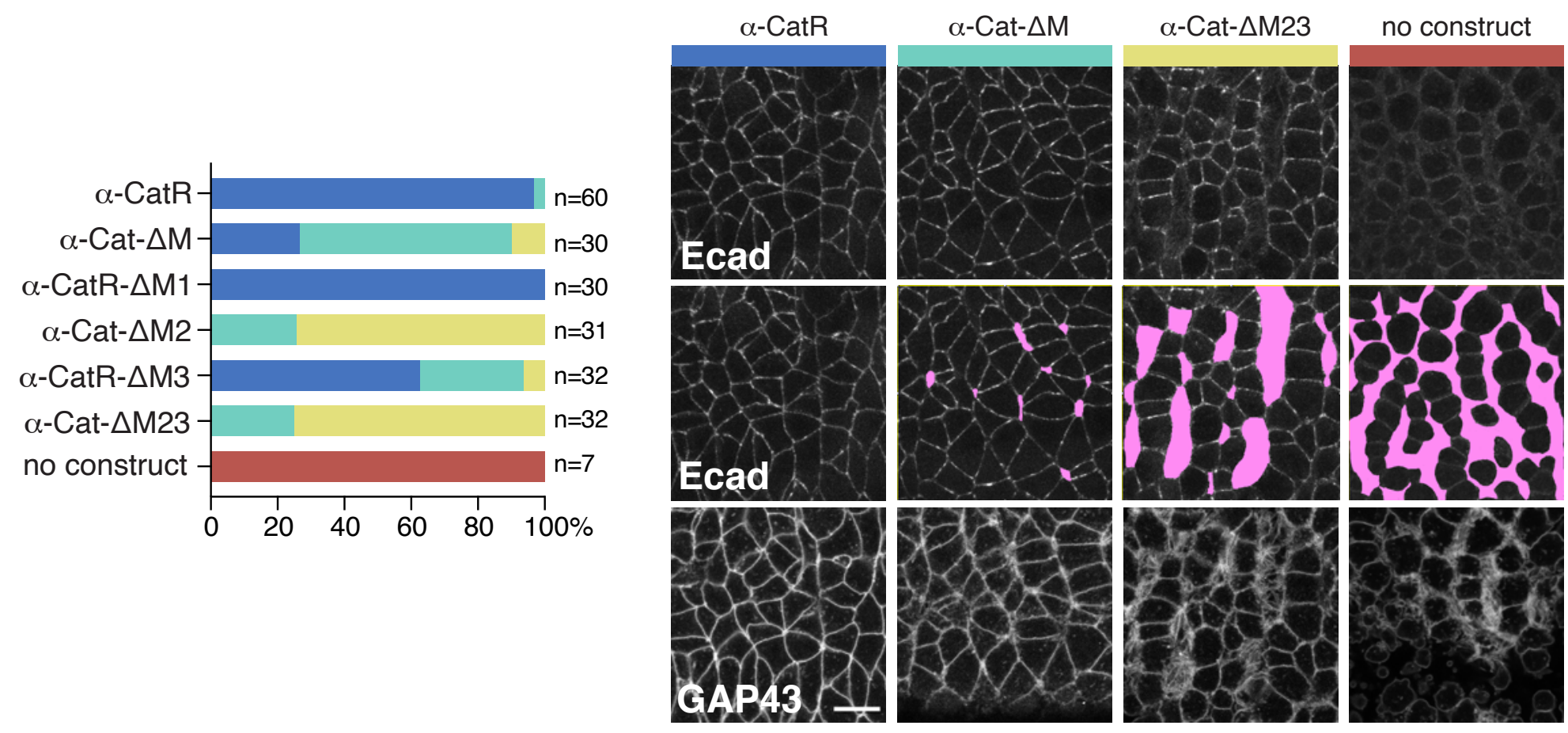

C Total area of gaps

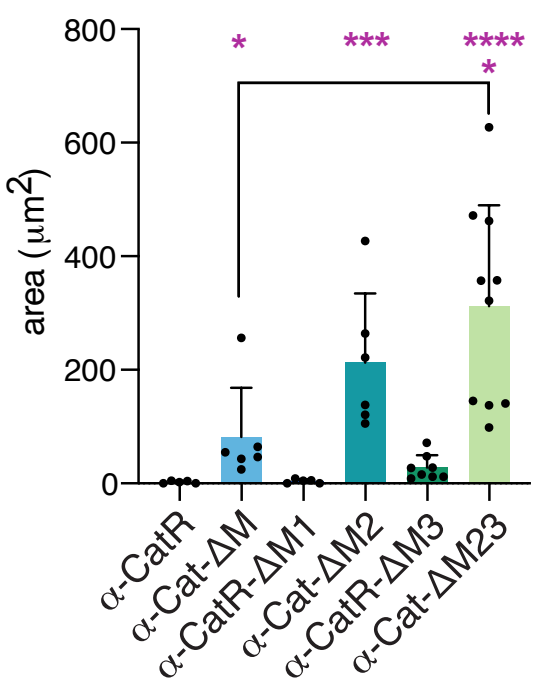

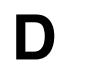

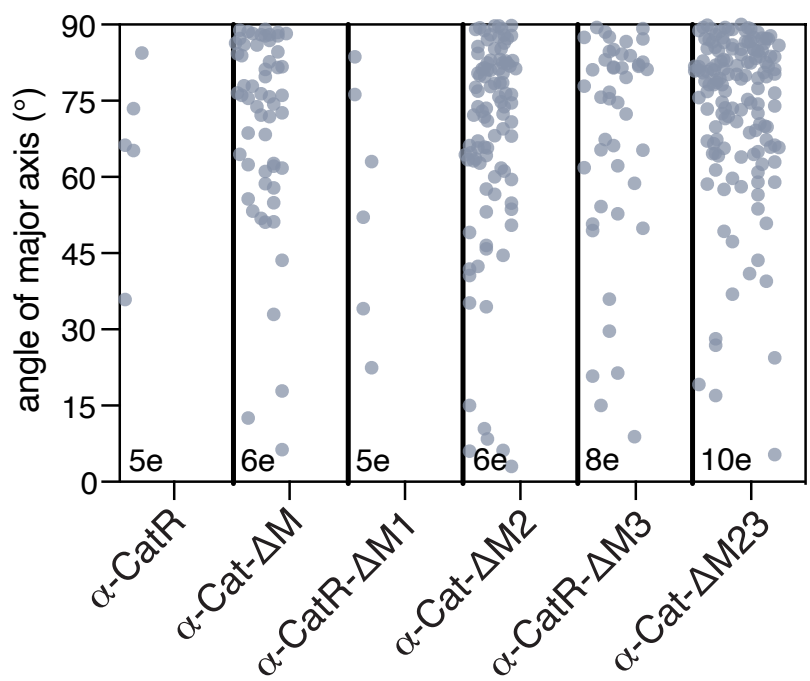

E $\quad{ }^{0.5} 7$ Germband extension

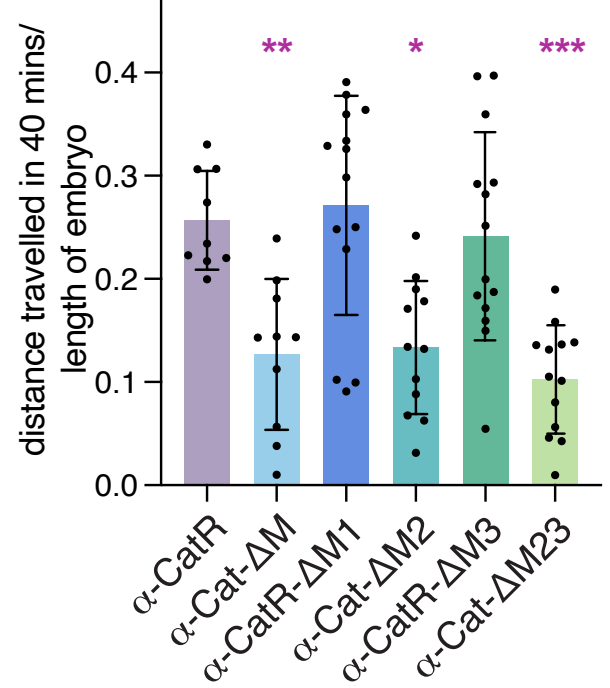


A $\alpha$-Cat-RNAi
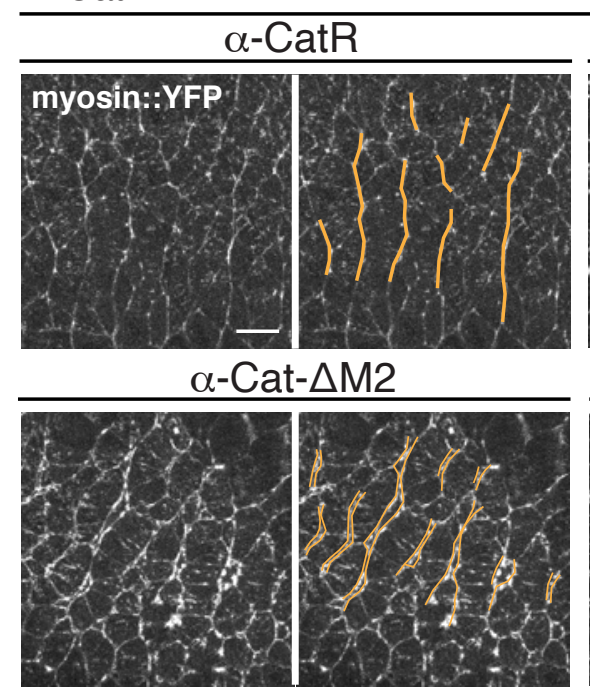

B

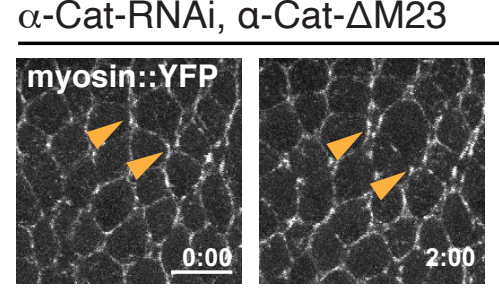

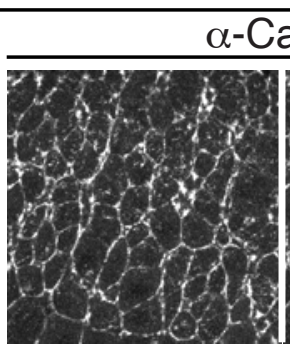

$\alpha-$ CatR- $\Delta \mathrm{M} 3$
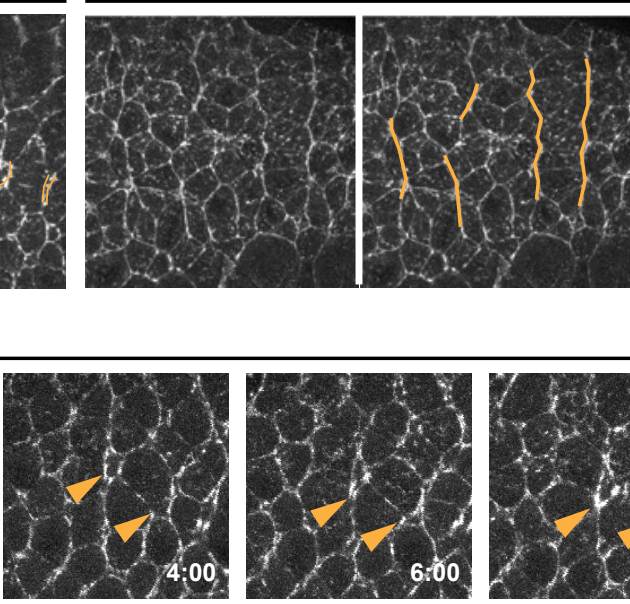

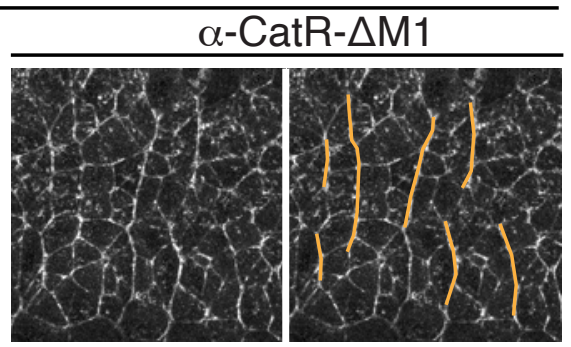

$\alpha$-Cat- $\Delta \mathrm{M} 23$

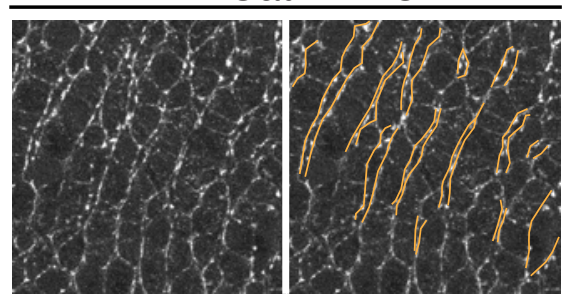

Time (mm:ss)
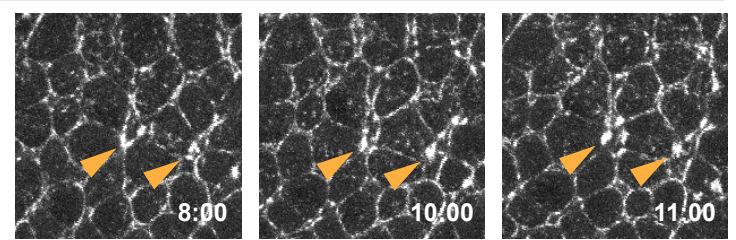

C
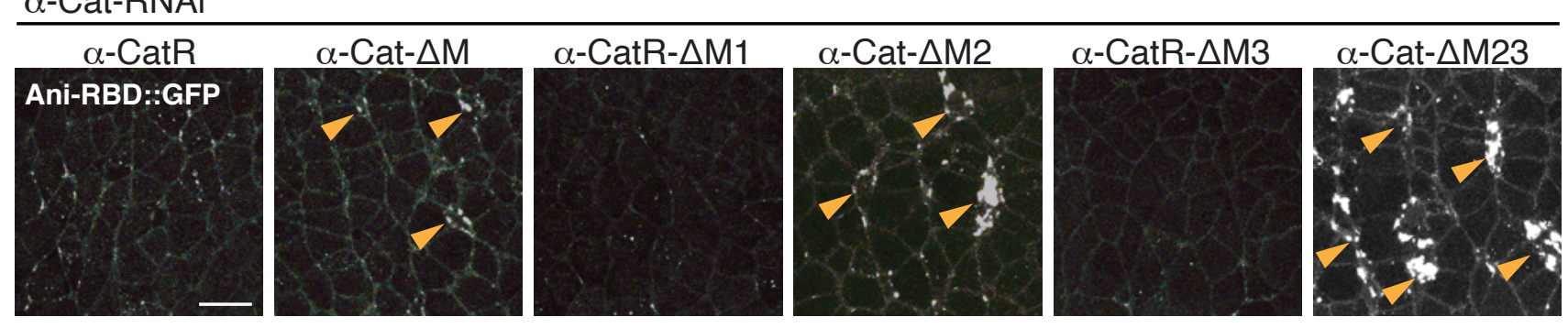

D
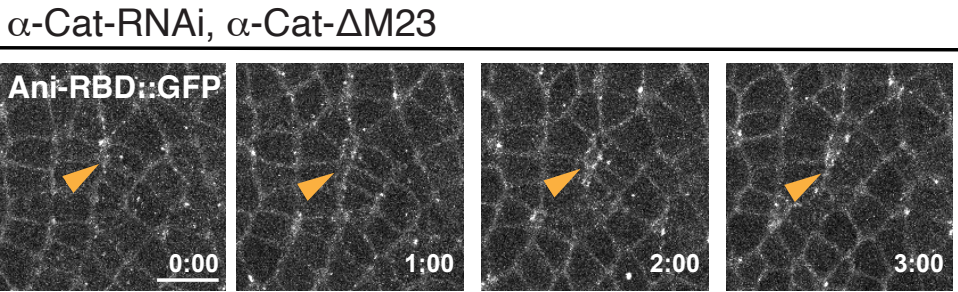

Time (mm:ss)
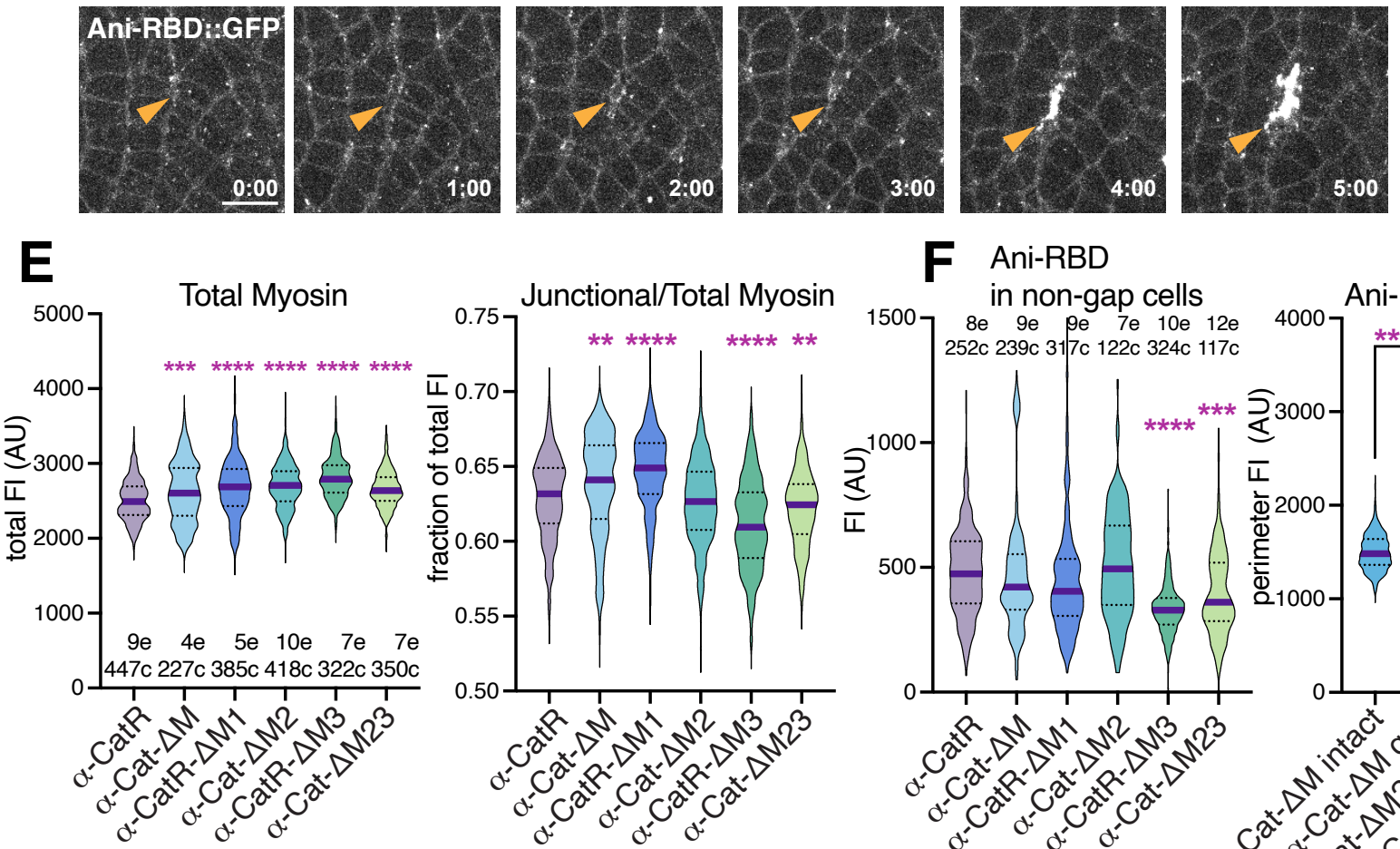

F Ani-RBD

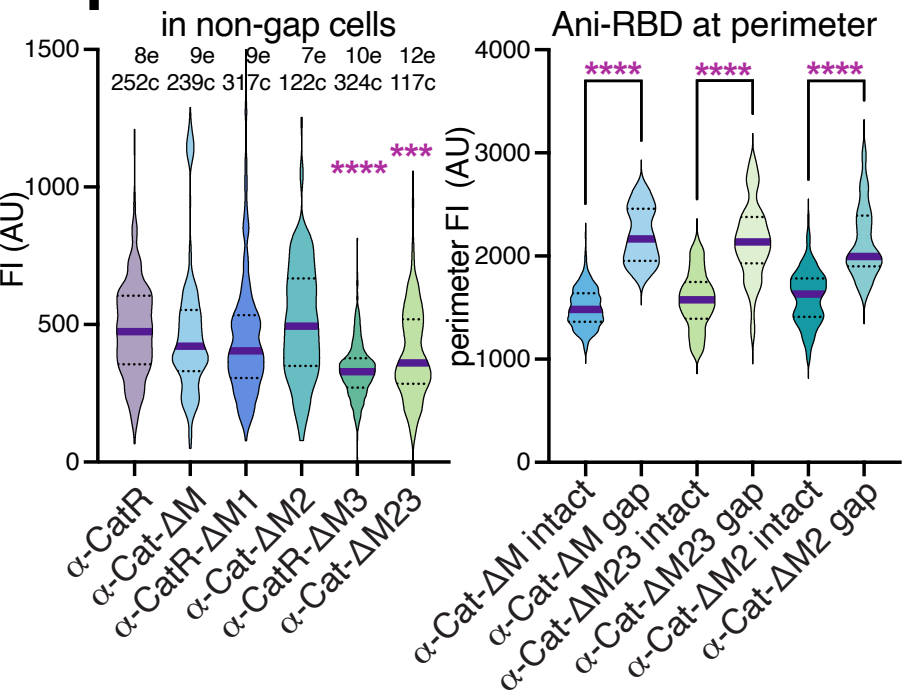

Sheppard \& Tepass, Figure 3 
bioRxiv preprint doi: https://doi.org/10.1101/2021.09.08.459511; this version posted September 9, 2021. The copyright holder for this preprint (which was not certified by peer review) is the author/funder, who has granted bioRxiv a license to display the preprint in perpetuity. It is made available under aCC-BY-NC-ND 4.0 International license.

A Vinc::mCherry, Ecad::GFP, $\alpha-C a t-R N A i$

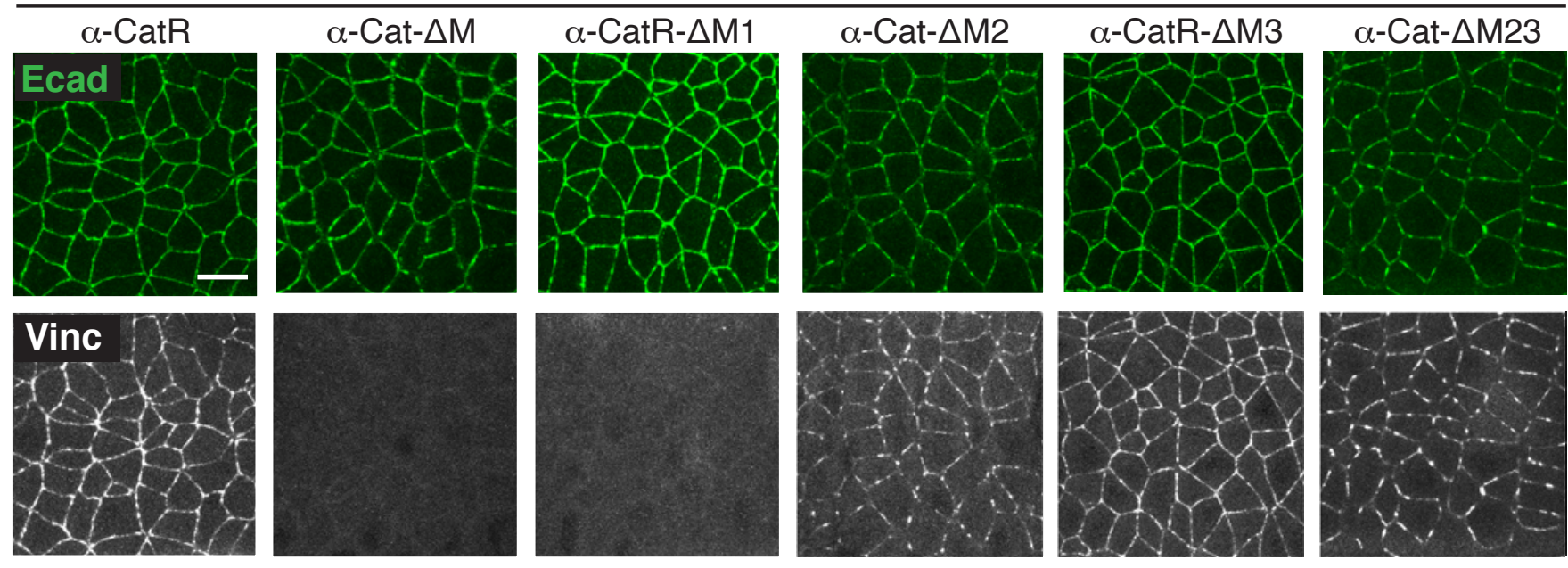

B

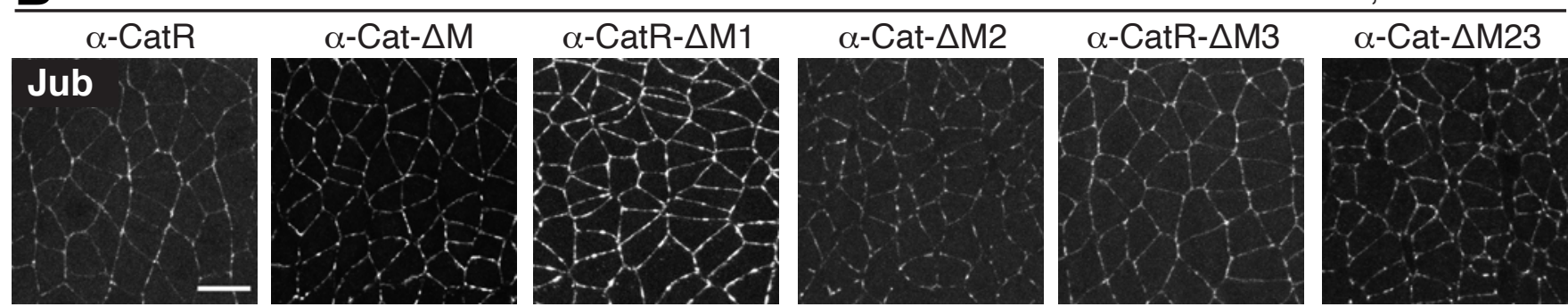

C
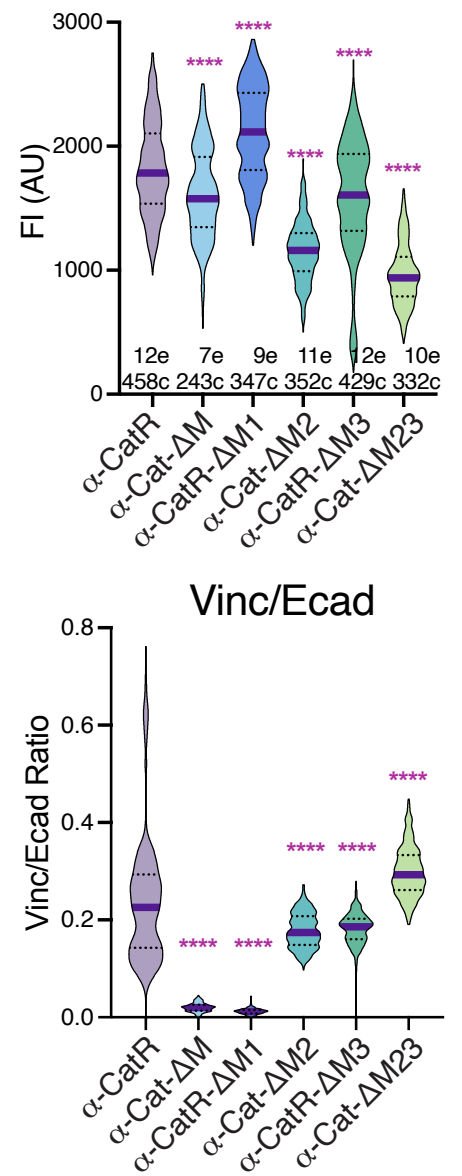

Vinc

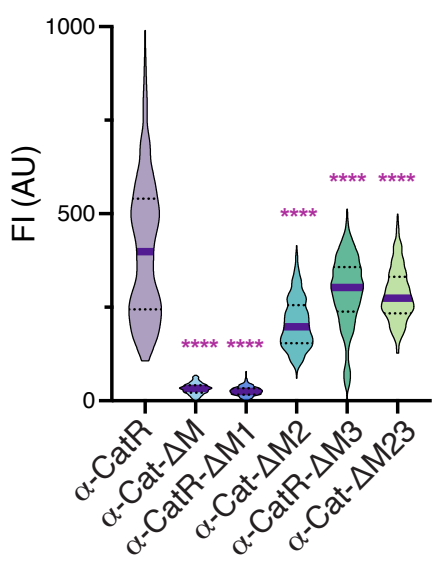

Jub

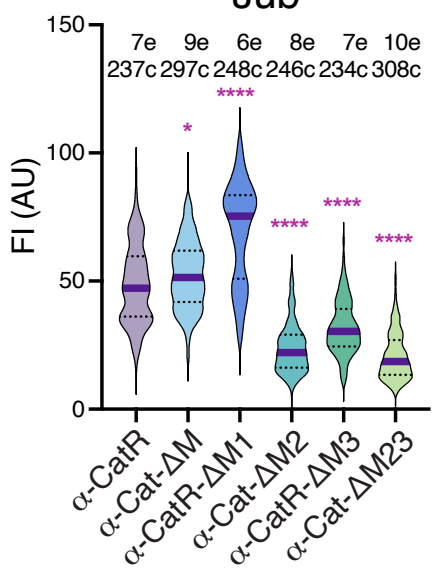

D Fold change in cortical levels versus $\alpha$-CatR

$<1 \quad=1 \quad>1$

Ecad Vinc Vinc/Ecad Jub

$\alpha-$ CatR

$\alpha-$ Cat- $\Delta \mathrm{M}$

$\alpha-$ CatR- $\Delta \mathrm{M} 1$

$\alpha-$ Cat- $\Delta \mathrm{M} 2$

$\alpha-$ CatR- $\Delta M 3$

$\alpha-$ Cat- $\Delta \mathrm{M} 23$

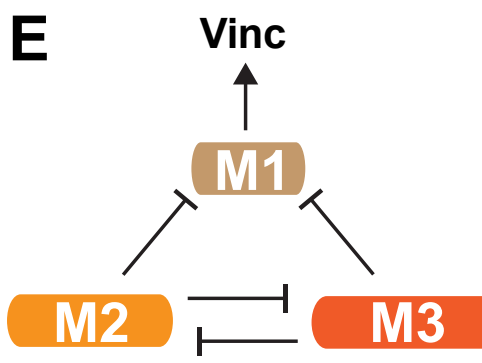



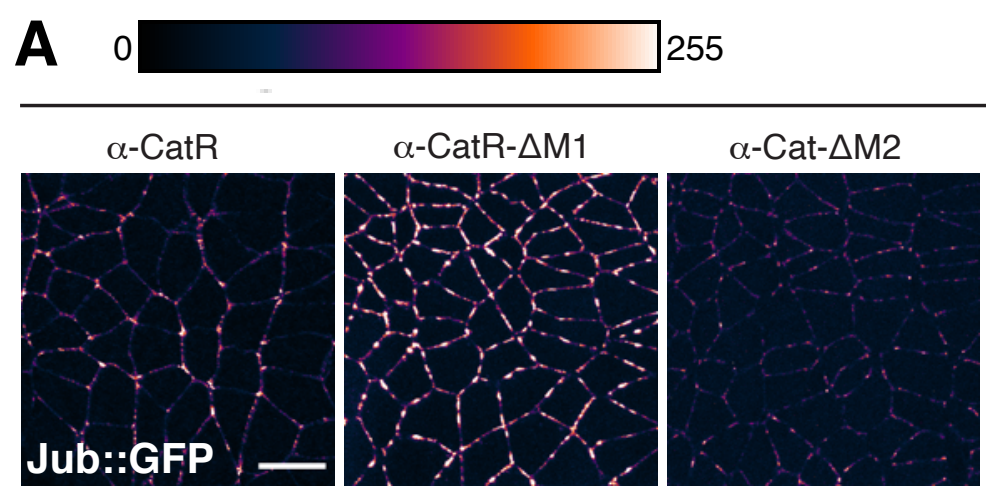

no construct

$\operatorname{DEcad} \Delta \beta:: \alpha-C a t-A B D$
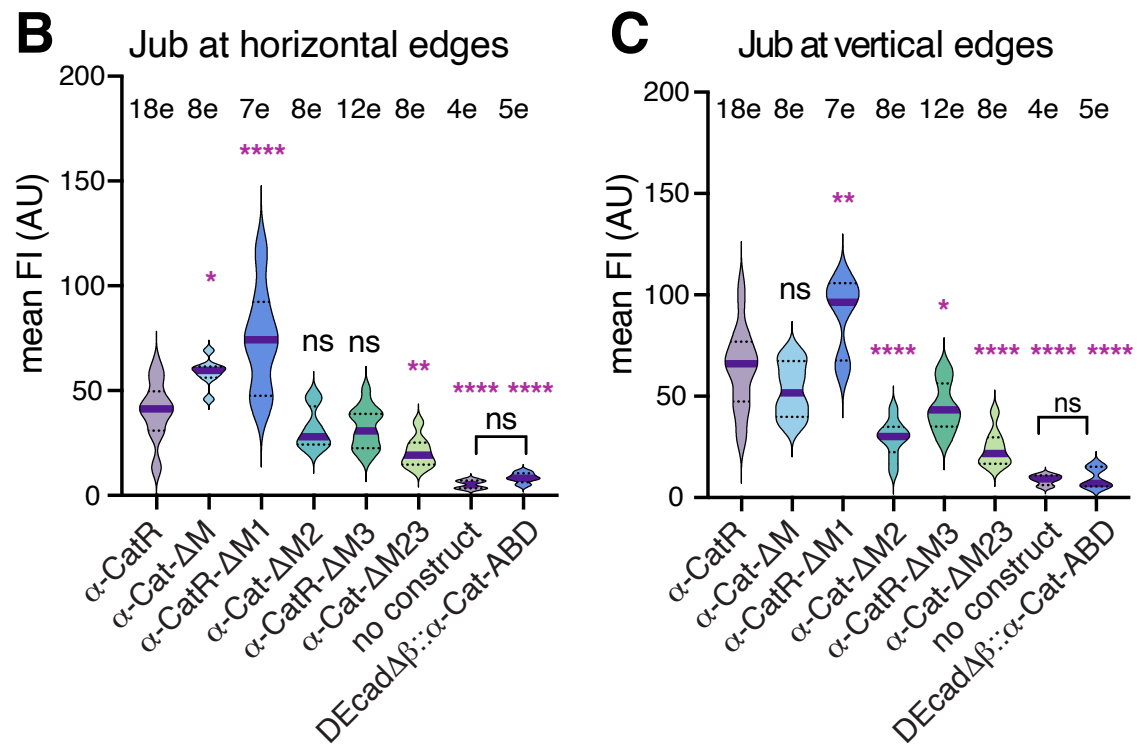

D Jub planar polarity
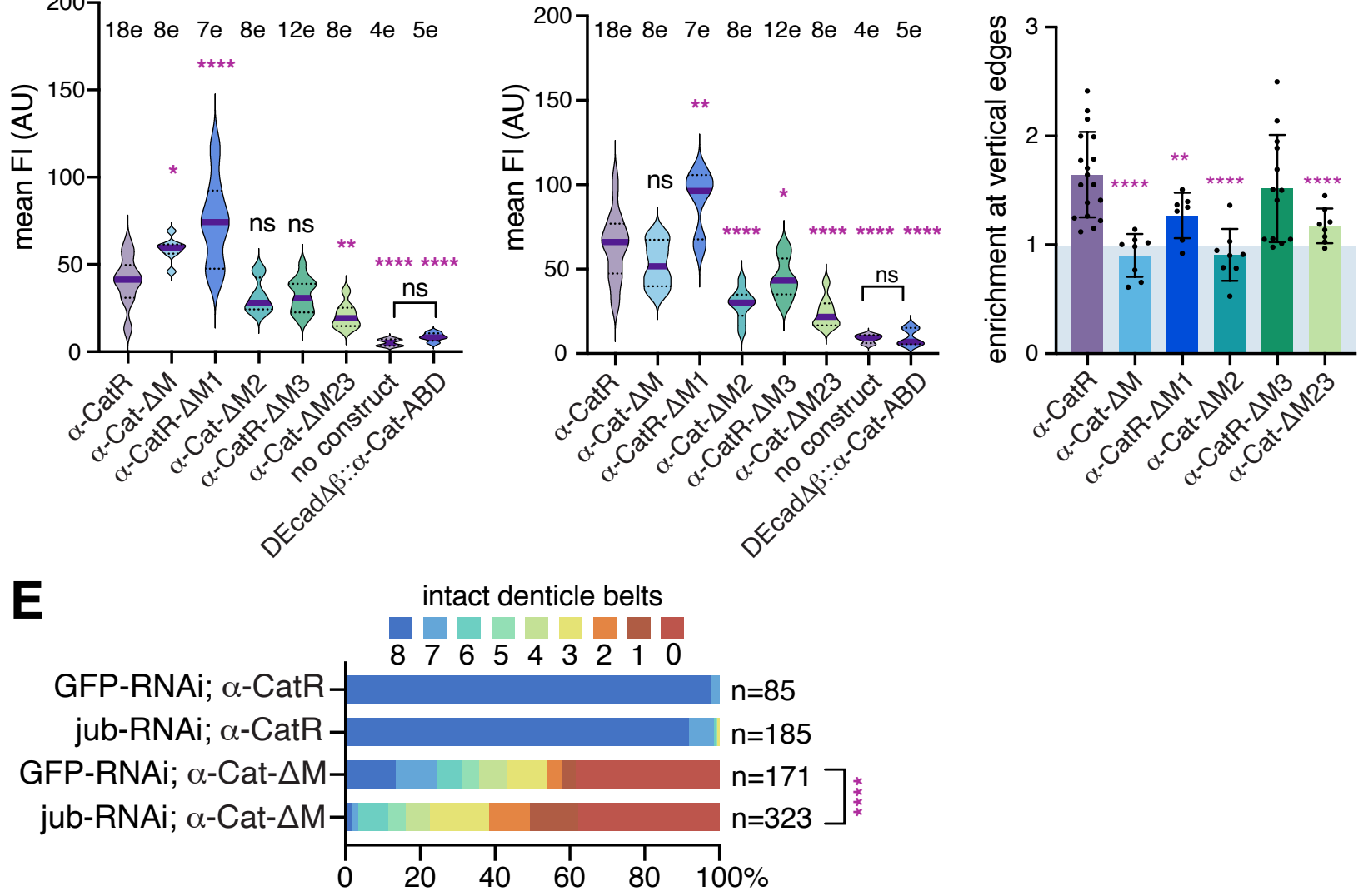

Sheppard \& Tepass, Figure 5 

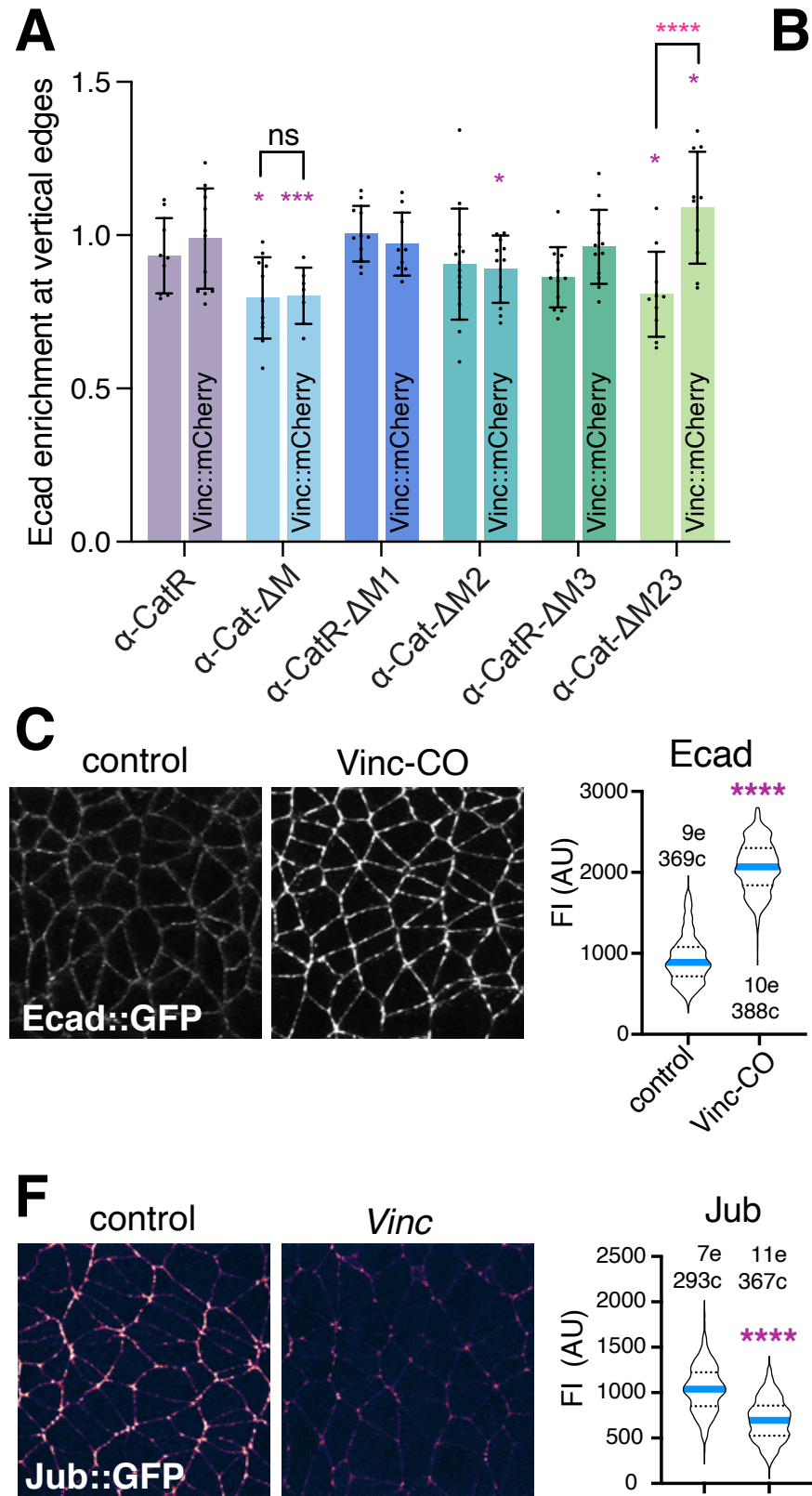

Vinc
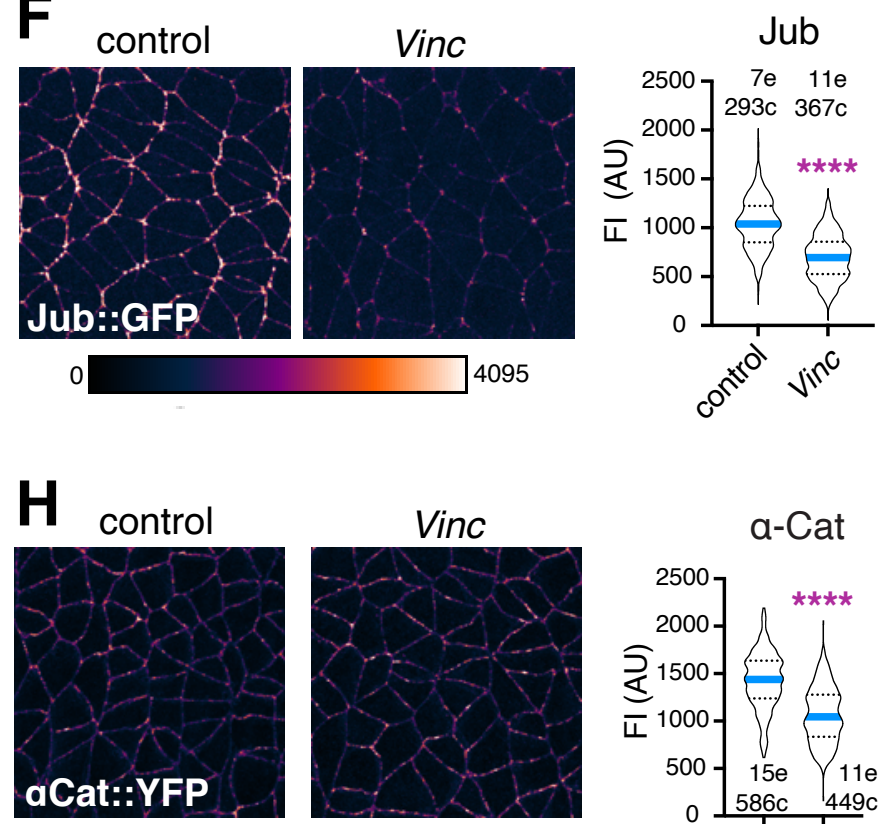

B
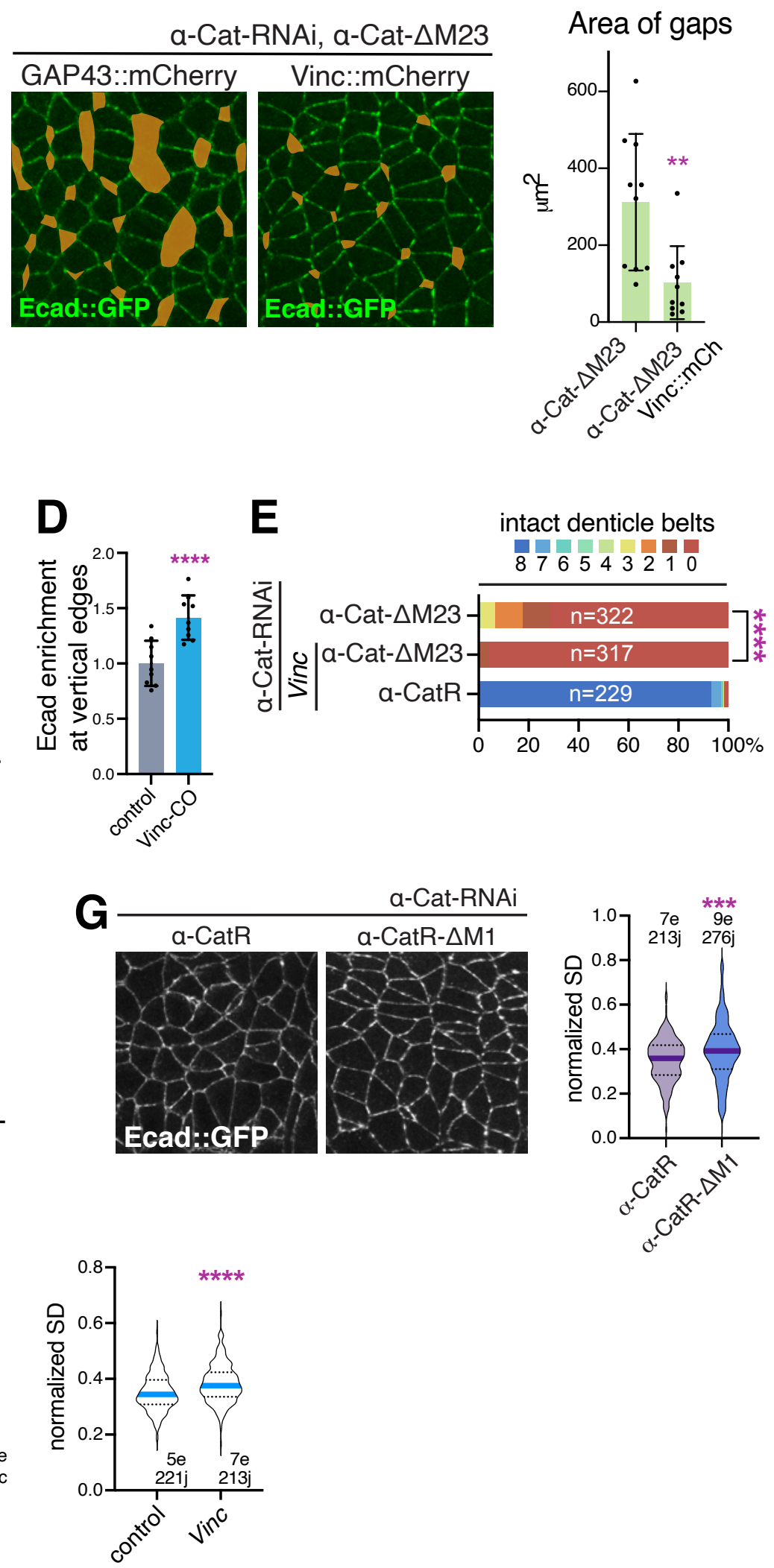

Sheppard \& Tepass, Figure 6 
bioRxiv preprint doi: https://doi.org/10.1101/2021.09.08.459511; this version posted September 9, 2021. The copyright holder for this preprint (which was not certified by peer review) is the author/funder, who has granted bioRxiv a license to display the preprint in perpetuity. It is made A available under aCC-BY-NC-ND 4.0 International license. $\alpha$-Cat-RNAi
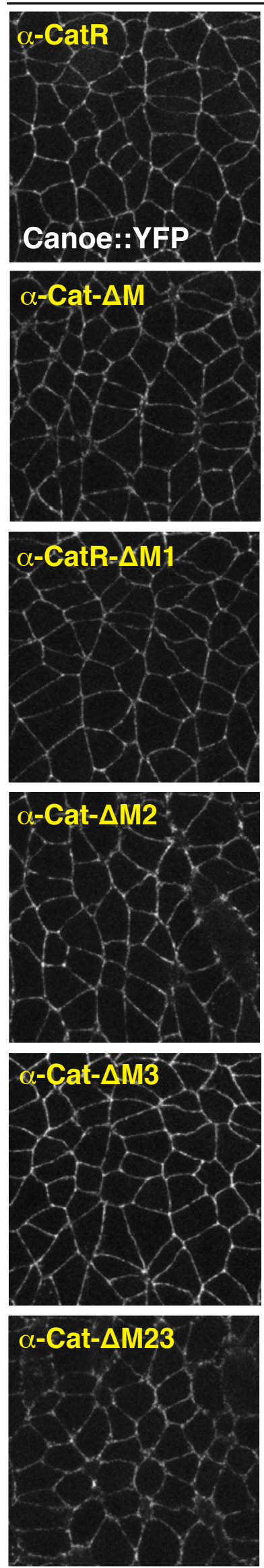
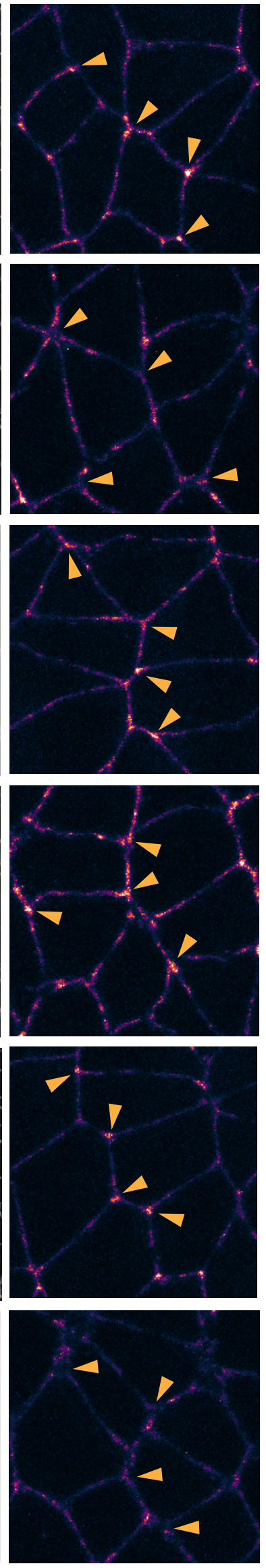
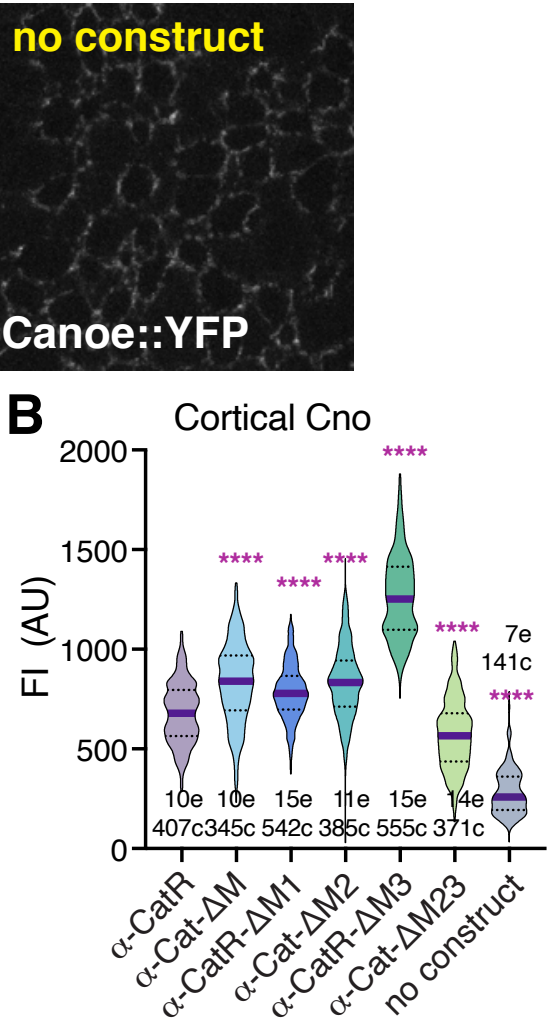

C

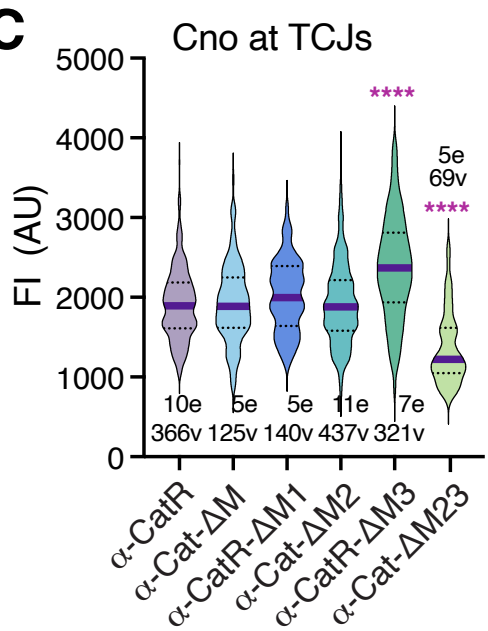

D Cno enrichment at TCJs/BJs

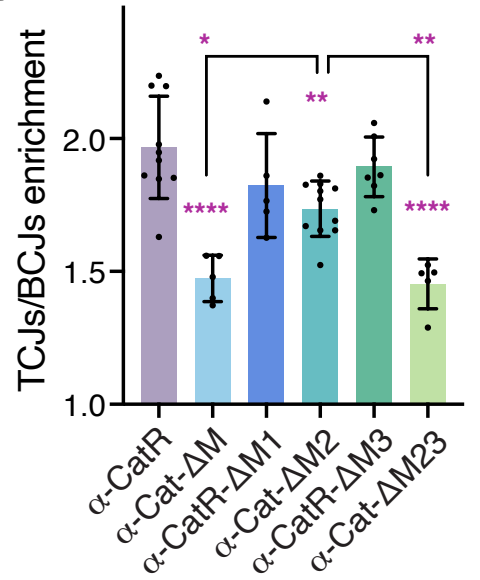



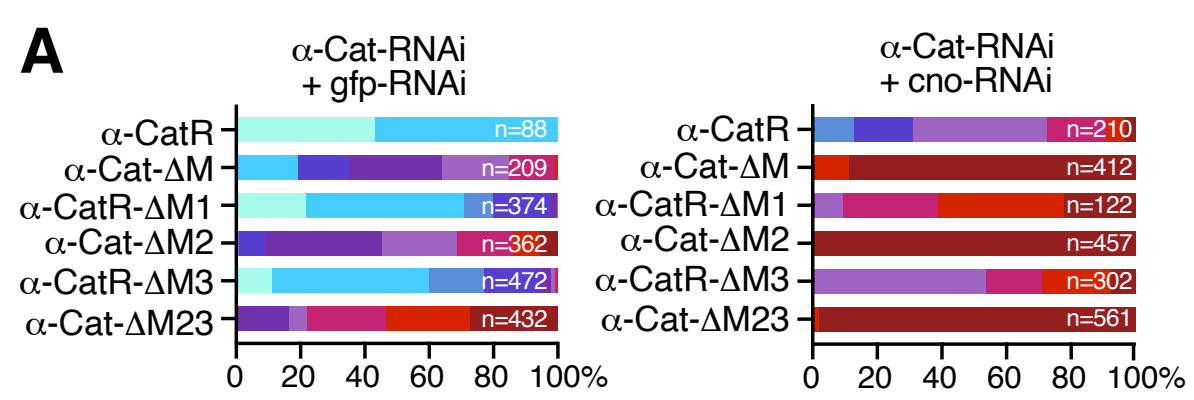

B

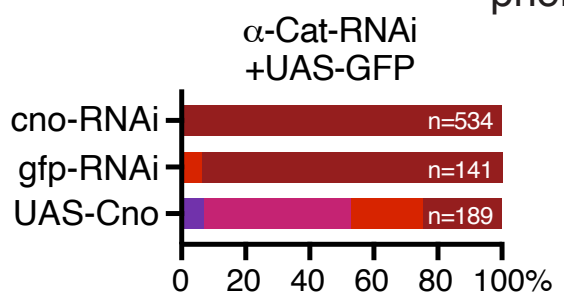

phenotypic category in $(A)$ and $(B)$

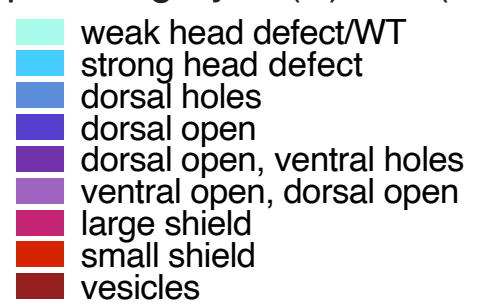

C

$\alpha$-Cat-RNAi + UAS-gfp-RNAi
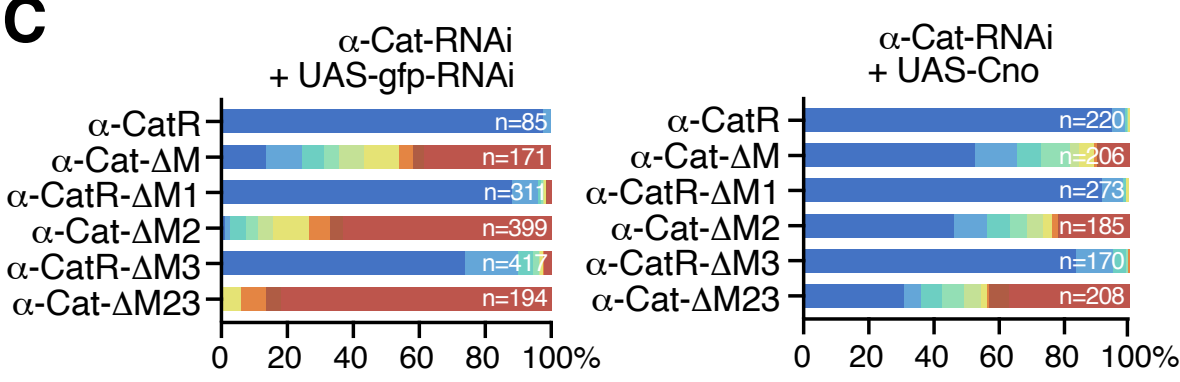

denticle belt counts in $(C),(E),(F)$, and $(G)$
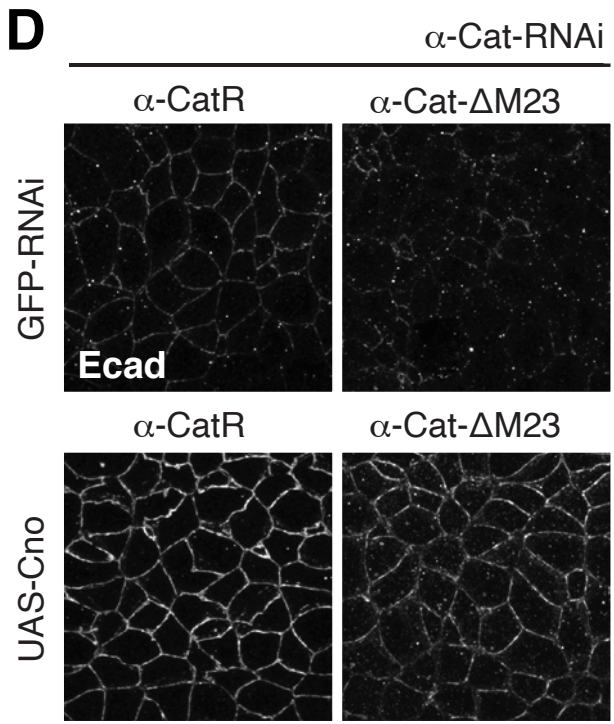

$\alpha-$ Cat- $\Delta \mathrm{M} 23$
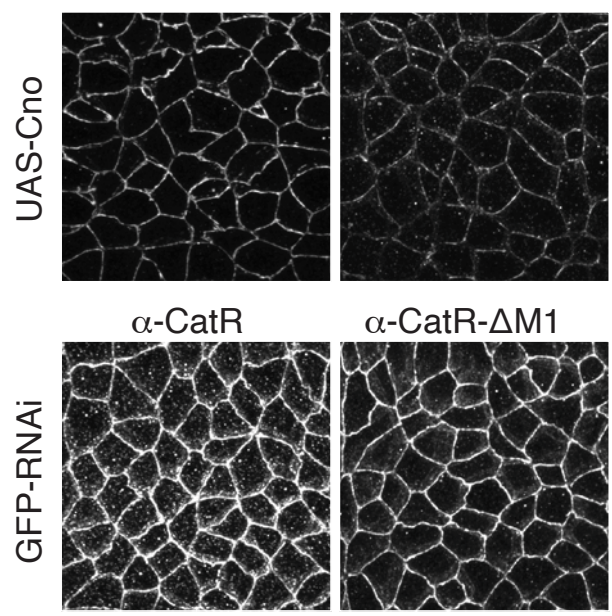

$\alpha-$ CatR- $\Delta \mathrm{M} 1$
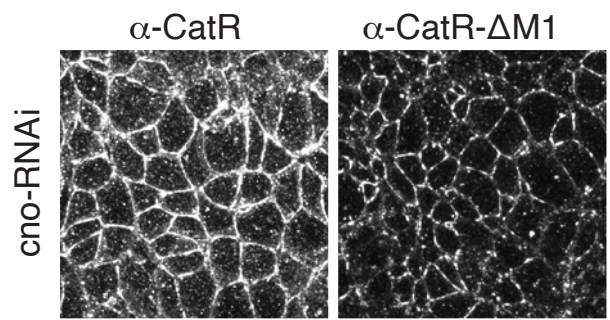

$\mathbf{E}$

$\mathbf{G}$

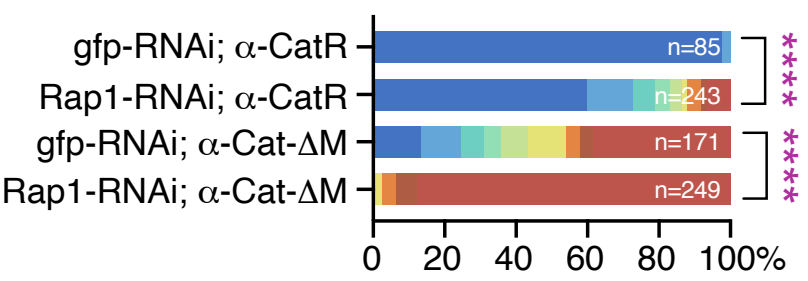

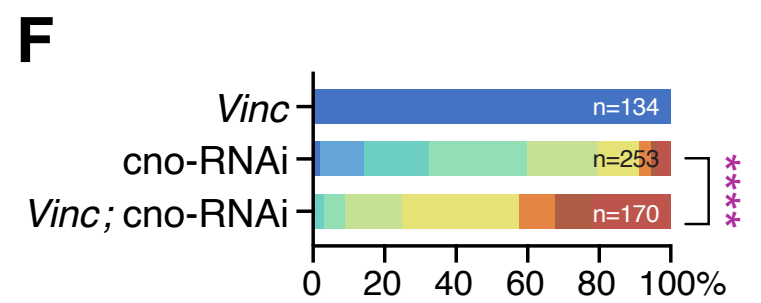

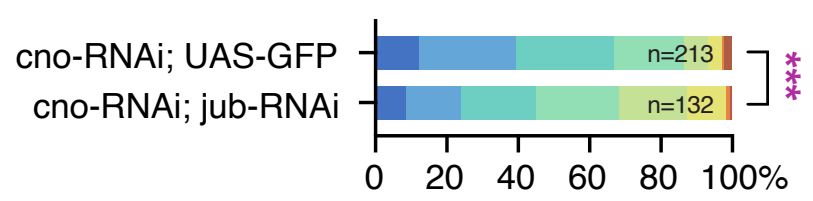




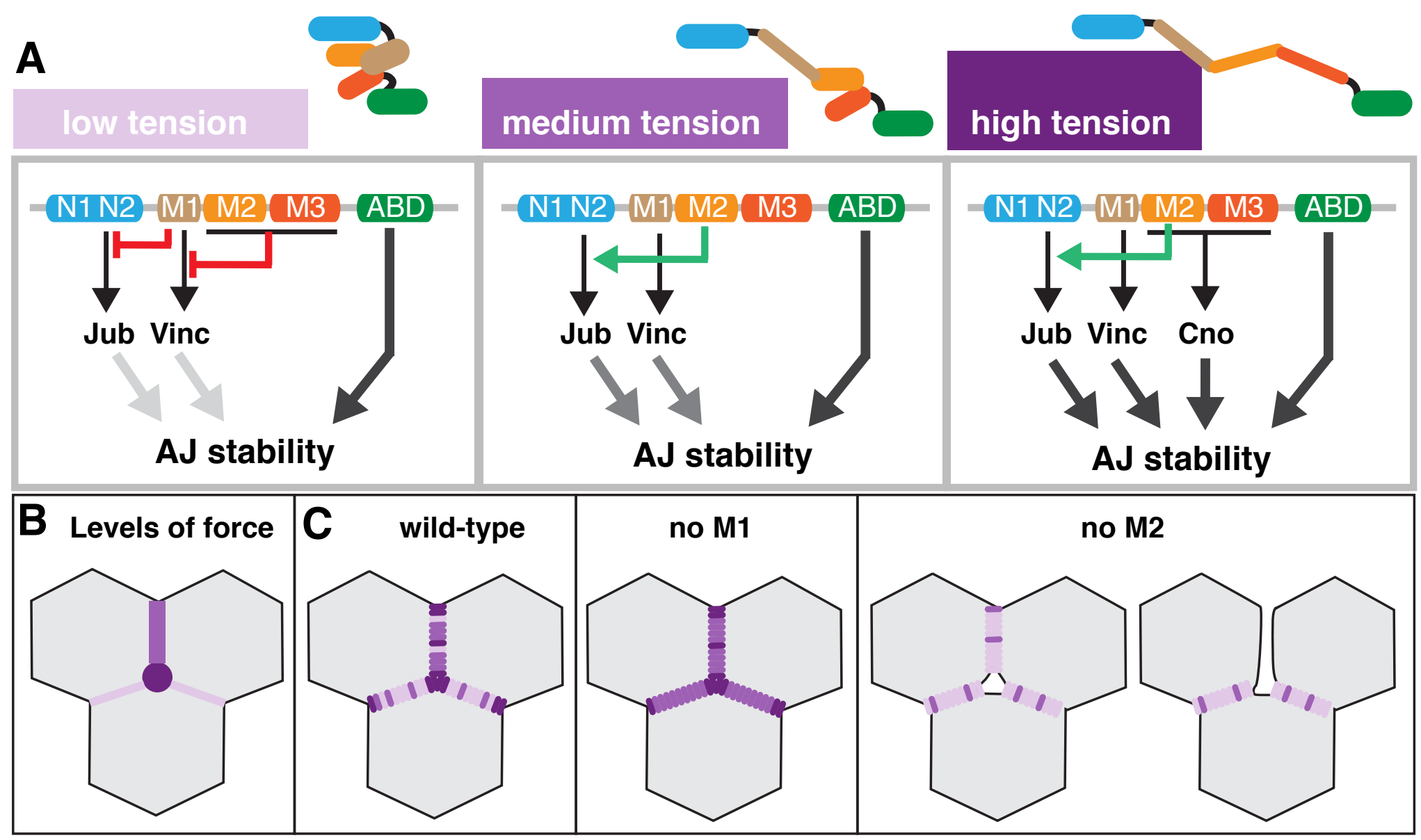

